\title{
LANGLANDS PARAMETERS OF DERIVED FUNCTOR MODULES AND VOGAN DIAGRAMS
}

\author{
PAUL D. FRIEDMAN
}

\begin{abstract}
Let $G$ be a linear reductive Lie group with finite center, let $K$ be a maximal compact subgroup, and assume that $\operatorname{rank} G=\operatorname{rank} K$. Let $\mathfrak{q}=\mathfrak{l} \oplus \mathfrak{u}$ be a $\theta$ stable parabolic subalgebra obtained by building $\mathfrak{l}$ from a subset of the compact simple roots and form $A_{\mathfrak{q}}(\lambda)$. Suppose $\Lambda=\lambda+2 \delta(\mathfrak{u} \cap \mathfrak{p})$ is $K$-dominant and the infinitesimal character, $\lambda+\delta$, of $A_{\mathfrak{q}}(\lambda)$ is nondominant due to a noncompact simple root. By interpreting these conditions on the level of Vogan diagrams, a conjecture by Knapp is (essentially) settled for the groups $G=S U(p, q), S p(p, q)$, and $S O^{*}(2 n)$, thereby determining the Langlands parameters of natural irreducible subquotient of $A_{\mathfrak{q}}(\lambda)$. For the remaining classical groups, simple supplementary conditions are given under which the Langlands parameters may be determined.
\end{abstract}

\section{Introduction}

Let $G$ be a linear reductive Lie group with finite center, and let $K$ be a maximal compact subgroup of $G$ corresponding to a global Cartan involution $\Theta$. Suppose that $\operatorname{rank} G=\operatorname{rank} K$, so that there is a maximal abelian subspace $t_{0}$ of $\mathfrak{f}_{0}$ that is also a Cartan subalgebra of $\mathfrak{g}_{0}$. Let $(\pi, V)$ be an admissible representation of $G$, that is, a representation in which each $K$-type occurs with only finite multiplicity in $\left.\pi\right|_{K}$. Work by Langlands [8], and subsequent work by Knapp and Zuckerman [7], parametrized irreducible admissible representations of $G$. The Langlands parameters of such a representation consist of a cuspidal parabolic subgroup $M A N$, a discrete series or a limit of discrete series on $M$, and a complex-valued linear functional on the Lie algebra of $A$ satisfying a positivity condition.

One approach to understanding a representation is to study it algebraically through its underlying ( $\mathfrak{g}, K)$ module. Theorems of Harish-Chandra, Lepowsky, and Rader show that every irreducible $(\mathfrak{g}, K)$ module is the underlying $(\mathfrak{g}, K)$ module of an irreducible admissible representation of $G$. Therefore, when one speaks of the Langlands parameters of an irreducible ( $(g, K)$ module $V$, one means those of an associated irreducible admissible representation of $G$. 
Let $(\mathfrak{g}, K)$ be a reductive pair, $\mathfrak{q}=\mathfrak{l} \oplus \mathfrak{u}$ be a $\theta$ stable parabolic subalgebra, $\mathrm{C}_{\lambda}$ be a one-dimensional ( $(\mathfrak{l}, L \cap K)$ module with weight $\lambda$, and consider the admissible cohomologically induced module $A_{\mathfrak{q}}(\lambda)$ (see [6] for details). When $L$ is compact and the infinitesimal character of $A_{\mathfrak{q}}(\lambda), \lambda+\delta$, lies in the "good zone," that is, has a positive inner product with the roots of $\mathfrak{u}$, then $A_{\mathfrak{q}}(\lambda)$ is in the discrete series of $G$. By allowing $\lambda+\delta$ to vary outside of the good zone, one produces "analytic continuations of discrete series" that are often nonzero irreducible unitarizable modules.

In [4], assuming primarily that $\Lambda=\lambda+2 \delta(\mathfrak{u} \cap \mathfrak{p})$ is $K$-dominant, $\mathfrak{l}$ is compact, and $A_{\mathfrak{q}}(\lambda)$ lies outside of the good zone due to a noncompact simple root, Knapp addressed the question of Langlands parameters of these analytically continued $A_{\mathfrak{q}}(\lambda)$ s (or of the unique irreducible subquotient of an $A_{\mathfrak{q}}(\lambda)$ containing the $K$-type with highest weight $\Lambda$ ). He proposed a simple recursive process for doing so, and conjectured that his process produces Langlands parameters. In certain cases, using combinatorial methods, he proved that his procedure worked.

The work in [2] studies Knapp's process and interprets it in the context of cohomological induction and intertwining operators. A key result of that paper gives a simple set of conditions on the roots in $\Delta^{+}(\mathfrak{g})$ and the infinitesimal character that when satisfied often allows one to validate Knapp's conjecture. These conditions were checked in [2] when the simple system of roots corresponding to $\Delta^{+}(\mathfrak{g})$ contained just one noncompact root.

This paper is primarily an investigation into the extent to which the techniques of [2] are able to resolve the conjecture in [4], allowing for more polarizations of $\mathfrak{q}=\mathfrak{l} \oplus \mathfrak{u}$. Under the equal rank hypothesis, we consider analytic continuations of discrete series modules, $A_{\mathfrak{q}}(\lambda)$, in which $\Upsilon$ is compact, $\Lambda$ is $K$-dominant, and the parameter $\lambda+\delta$ has varied into a range where it is nondominant due to noncompact simple roots. (The requirement that $\mathbb{l}$ is compact is not major, as Corollary 11.229 of [6] in essence reduces consideration to this case.) We show in Theorem 4.1 that for the groups $S U(p, q), S p(p, q)$, and $S O^{*}(2 n)$, the techniques of [2] work, and Knapp's conjecture is (essentially) settled. For the remaining two classes of classical groups satisfying the equal rank hypothesis, namely $S O(p, q)$ with $p$ and $q$ not both odd, and $S p(n, \mathbf{R})$, the methods of [2] are NOT enough. For these groups, in Theorems 4.6 and 4.7, we try to develop additional hypotheses under which [2] resolves the conjecture in [4]. Theorem 4.7 provides a reasonable partial solution. Assuming that $\lambda+\delta$ has a sufficiently positive inner product versus one distinguished root of $\Delta^{+}(\mathfrak{g})$, the groups $S O(p, q)$ are handled. For $S p(n, \mathrm{R})$, unfortunately, we need to place a further restriction on $\lambda+\delta$.

Further, in Theorem 4.9, we determine the extent of which the conditions in [2] hold when $A_{\mathfrak{q}}(\lambda)$ is in the "weakly fair" zone. These modules are of 
particular interest for a number of reasons; in particular, results of Vogan show they are unitarizable, and when $q$ is of "symmetric-type," they are the underlying modules of the Flensted-Jensen discrete series.

This paper is organized as follows. Section 2 contains the preliminary information; in it we describe the conjectured method of [4], give the reduced criteria of [2] that we will use to check Knapp's process, and codify the information contained in a Vogan diagram into calculation ready notation. Section 3 provides some simple but powerful computional lemmas. Section 4 contains the main results, and Section 5 contains a collection of the computational proofs.

\section{Preliminaries}

\subsection{Basic Notational Conventions}

Let $G$ be a reductive Lie group with finite center and let $K$ be a maximal compact subgroup. We will always assume that $\operatorname{rank} G=\operatorname{rank} K$. We denote Lie algebras of a Lie group by the corresponding Gothic letters with subscripts 0 , and we denote complexifications by dropping the subscripts. We let bar denote the conjugation of $g$ with respect to $\mathfrak{g}_{0}$. Let $\theta$ be the Cartan involution of $\mathfrak{g}_{0}$ corresponding to $K$ and let $\mathfrak{g}_{0}=\mathfrak{f}_{0} \oplus \mathfrak{p}_{0}$ be the associated Cartan decomposition.

Let $\mathrm{t}_{0}$ be a $\theta$ stable Cartan subalgebra of $\mathfrak{f}_{0}$ and therefore of $\mathfrak{g}_{0}$, and let $\Delta=\Delta(\mathrm{g}, \mathrm{t})$ be the set of roots. We introduce in the usual way an inner product $\langle\cdot, \cdot\rangle$ and a norm squared $|\cdot|^{2}$ on the real linear span of the roots. We use a hat to denote a coroot; that is, if $\alpha \in \Delta$ then $\hat{\alpha}=2 \alpha /|\alpha|^{2}$. Since the Cartan subalgebra lies in $\mathfrak{f}_{0}$, each root vector lies in $\mathfrak{f}$ or in $\mathfrak{p}$, and the roots are called compact or noncompact accordingly. We denote the subset of compact roots by $\Delta_{K}$ and the subset of noncompact roots by $\Delta(\mathfrak{p})$.

Let $\mathfrak{q}=\mathfrak{l} \oplus \mathfrak{u}$ be the $\theta$ stable parabolic subalgebra with $\mathfrak{l}$ formed from a subset of the compact simple roots, and $\mathfrak{u}$ formed from the remaining positive roots. Let $\lambda$ be an analytically integral linear functional on that is orthogonal to all members of $\Delta(\mathfrak{l})$, and with $\langle\lambda, \beta\rangle \geq 0$ for all other simple compact roots. Let $\mathrm{C}_{\lambda}$ be the one-dimensional $(\mathfrak{l}, L \cap K)$ module with highest weight $\lambda$. Let $A_{\mathfrak{q}}(\lambda)$ be the Zuckerman $(\mathfrak{g}, K)$ module with infinitesimal character $\lambda+\delta$ defined (as in [6]) by

$$
A_{\mathfrak{q}}(\lambda)=\mathscr{L}_{S}\left(\mathrm{C}_{\lambda}\right)
$$

Since $L$ is compact, one can combine an algebraic Borel-Weil theorem with an induction-in-stages result to show that $A_{\mathfrak{q}}(\lambda) \cong A_{\mathfrak{b}}(\lambda)$, where $\mathfrak{b}$ is a Borel subalgebra contained in $\mathfrak{q}$. Let $\Lambda=\lambda+2 \delta(\mathfrak{H} \cap \mathfrak{p})=\lambda+2 \delta(\mathfrak{p})=(\lambda+\delta)+(\delta-$ $\left.2 \delta_{K}\right)$. Throughout this paper, we shall always assume that $\Lambda$ is $\Delta_{K}^{+}$-dominant so that it occurs with multiplicity one in $A_{\mathfrak{q}}(\lambda)$. 


\subsection{Langlands Parameters}

The Langlands classification of irreducible admissible representations of $G$ is well known (see, for example, [3, Th. 14.91]) and by the Langlands parameters of an irreducible $(g, K)$ module $V$ we mean a triple $(M A N, \sigma, v)$ such that

(1) $M A N$ is a cuspidal parabolic subgroup of $G$

(2) $\sigma$ is a discrete series or limit of discrete series on $M$ with infinitesimal character $\lambda_{\sigma}$

(3) $v$ is a complex-valued linear functional on the Lie algebra $a_{0}$ of $A$ with Re $v$ in the closed positive Weyl chamber

(4) the induced representation $\operatorname{ind}_{M A N}^{G}\left(\sigma \otimes e^{v} \otimes 1\right)$ has a unique irreducible quotient, called the Langlands quotient and denoted $J(M A N, \sigma, v)$

(5) $V$ is equivalent with the underlying $(g, K)$ module of $J(M A N, \sigma, v)$.

As stated above, the Langlands parameters are not unique.

\subsection{The Knapp Process}

In this section we describe Knapp's conjectural method [4] for determining the Langlands parameters of the subquotient of $A_{\mathfrak{q}}(\lambda)$ containing the $K$-type with highest weight $\Lambda$. Roughly, if the infinitesimal character $\lambda+\delta$ of $A_{\mathfrak{q}}(\lambda)$ is nondominant versus a noncompact root $\alpha$, then split, by the Cayley transform relative to $\alpha$, the Cartan subalgebra $\mathrm{t}$ into $\mathrm{t}^{\prime} \oplus \mathrm{a}^{\prime}$. Project the infinitesimal character onto the dual of each of these pieces, but negate the projection onto the $\mathfrak{a}^{\prime}$ piece. Label these projections $\lambda_{\sigma^{\prime}}$ and $v$. Form $M^{\prime}=Z_{G}\left(\mathfrak{a}^{\prime}\right)$ and the roots $\Delta^{+}\left(\mathfrak{m}^{\prime}, \mathfrak{t}^{\prime}\right)$, which may be identified with the roots of $\Delta^{+}(\mathfrak{g})$ orthogonal to $\alpha$. As shown in [4], the functional $\lambda_{\sigma^{\prime}}$ will be dominant versus the compact simple roots of $\Delta^{+}\left(\mathfrak{m}^{\prime}\right)$ and the corresponding weight $\Lambda^{\prime}$ will be $M^{\prime} \cap K$ dominant. Thus, one may continue this process on $M^{\prime}$ and the corresponding $A_{\mathfrak{q}_{\mathfrak{m}^{\prime}}}\left(\lambda^{\prime}\right)$ with infinitesimal character $\lambda_{\sigma^{\prime}}$, increasing the dimension of $\mathfrak{a}$ at each step until a discrete series module is produced on a subsequent $M$.

More precisely, set $M_{0}=G, A_{0}=\{I\}, \mathfrak{t}_{0}^{0}=\mathfrak{t}_{0}, \mathfrak{a}_{0}^{0}=0, \mathfrak{h}_{0}^{0}=\mathfrak{t}_{0}^{0} \oplus \mathfrak{a}_{0}^{0}$, $\lambda_{0}=\lambda, \delta_{0}=\delta, \lambda_{\sigma_{0}}=\lambda_{0}+\delta_{0}, v_{0}=0, \Lambda_{0}=\lambda_{\sigma_{0}}+\left(\delta_{0}-2 \delta_{0, K}\right)$. Suppose $M_{j}, A_{j}, \mathfrak{t}_{0}^{j}, \mathfrak{a}_{0}^{j}, \mathfrak{h}_{0}^{j}, \lambda_{j}, \delta_{j}, \lambda_{\sigma_{j}}, \Lambda_{j}$ and $v_{j}$ are given with $\operatorname{dim} A_{j}=j$ and with $\lambda_{\sigma_{j}}$ dominant nonsingular with respect to all simple roots of $M_{j}$ that are $M_{j}$ compact. There are now two cases:

(a) If $\left\langle\lambda_{\sigma_{j}}, \alpha\right\rangle \geq 0$ for all simple roots $\alpha$ of $M_{j}$ that are $M_{j}$ noncompact, the recursive construction ends. Define $M=M_{j}, A=A_{j}, \lambda_{\sigma}=\lambda_{\sigma_{j}}$, and $v=v_{0}+\cdots+v_{j}$. Define $N$ so that $v$ is dominant relative to $N$. Then $M A N, \lambda_{\sigma}$, and $v$ are the cuspidal parabolic subgroup, the infinitesimal character of the $M$ representation, and the parameter on $\mathfrak{a}_{0}$ of a set of Langlands parameters for the irreducible subquotient of $A_{\mathfrak{q}}(\lambda)$ containing the $K$-type $\Lambda$. 
(b) Otherwise, of the $M_{j}$ noncompact simple roots $\alpha$ with $\left\langle\lambda_{\sigma_{j}}, \alpha\right\rangle<0$, set $\alpha_{j+1}$ to be one for which $-\left\langle\lambda_{\sigma_{j}}, \hat{\alpha}\right\rangle$ is greatest. Further, set

$$
c_{j+1}=-\frac{\left\langle\lambda_{\sigma_{j}}, \alpha_{j+1}\right\rangle}{\left|\alpha_{j+1}\right|^{2}}=\frac{\left\langle s_{\alpha_{j+1}}\left(\lambda_{\sigma_{j}}\right), \alpha_{j+1}\right\rangle}{\left|\alpha_{j+1}\right|^{2}}
$$

where $s_{\alpha_{j+1}}$ is the Weyl group reflection corresponding to $\alpha_{j+1}$. Applying the Cayley transform relative to $\alpha_{j+1}$ we write $\mathfrak{h}_{0}^{j+1}=\mathrm{t}_{0}^{j+1} \oplus \mathfrak{a}_{0}^{j+1}$ for the transformed version of $\mathfrak{h}_{0}^{j}$ and let $A_{j+1}=\exp \left(\mathfrak{a}_{0}^{j+1}\right)$ with $\operatorname{dim} A_{j+1}=j+1$. Identifying $\alpha_{j+1}$ with its Cayley transform, set

$$
v_{j+1}=c_{j+1} \alpha_{j+1} \quad \text { and } \quad v^{j+1}=v^{j}+v_{j+1} .
$$

Define $N_{j+1}$ so that $v^{j+1}$ is dominant relative to $N_{j+1}$. Let $M_{j+1} A_{j+1}=$ $Z_{G}\left(A_{j+1}\right)$, and we identify $\Delta\left(\mathfrak{m}_{j+1}, \mathrm{t}^{j+1}\right)$ with the subset of $\Delta\left(\mathfrak{m}_{j}, \mathrm{t}^{j}\right)$ orthogonal to $\alpha_{j+1}$. Set $\Delta_{M_{j+1}}^{+}=\Delta_{M_{j+1}} \cap \Delta_{M_{j}}^{+}$, let $\delta_{j+1}$ be half the sum of the positive roots and $\delta_{j+1, K}$ be half the sum of the positive $M_{j+1}$ compact roots. Define $\lambda_{\sigma_{j+1}}$ to be the projection of $\lambda_{\sigma_{j}}$ orthogonal to $\alpha_{j+1}$, so that

$$
\begin{aligned}
\lambda_{\sigma_{j+1}} & =\lambda_{\sigma_{j}}-\frac{\left\langle\lambda_{\sigma_{j}}, \alpha_{j+1}\right\rangle}{\left|\alpha_{j+1}\right|^{2}} \alpha_{j+1}=\lambda_{\sigma_{j}}+\frac{\left\langle s_{\alpha_{j+1}}\left(\lambda_{\sigma_{j}}\right), \alpha_{j+1}\right\rangle}{\left|\alpha_{j+1}\right|^{2}} \alpha_{j+1} \\
& =\lambda_{\sigma_{j}}+c_{j+1} \alpha_{j+1}=\lambda_{\sigma_{j}}+v_{j+1} .
\end{aligned}
$$

We also define $\lambda_{j+1}$ so that $\lambda_{\sigma_{j+1}}=\lambda_{j+1}+\delta_{j+1}$ and set $\Lambda_{j+1}=\lambda_{\sigma_{j+1}}+\left(\delta_{j+1}-\right.$ $2 \delta_{j+1, K}$ ). Then $\lambda_{\sigma_{j+1}}$ is dominant nonsingular relative to the $M_{j+1}$ compact simple roots, and the recursive construction continues.

Proposition 10 of [4] shows that the Conjectural Method runs into no obstruction in finding parameters $M A N, \lambda_{\sigma}$, and $v$. In particular,

(1) $\lambda_{j}$ is analytically integral, and

(2) $\Lambda_{j}$ is $\Delta_{M_{j, K}}^{+}$dominant.

Finally we isolate two conditions built into Knapp's process. If $\alpha$ is the noncompact simple root chosen by the process at a particular step, and $\beta$ is another noncompact simple root at that step, then

$$
\begin{aligned}
& \langle\lambda+\delta, \hat{\beta}\rangle \geq\langle\lambda+\delta, \hat{\alpha}\rangle \\
& \langle\lambda+\delta, \hat{\alpha}\rangle \in \mathbf{Z}_{\leq-1}
\end{aligned}
$$




\subsection{Reduction of Problem}

The principal results in [2] concern the determination of conditions under which Knapp's method does in fact give a set of Langlands parameters of $V$, the subquotient of $A_{\mathfrak{q}}(\lambda)$ containing the $K$-type with highest weight $\Lambda$. The results hinge on [2, Theorem 5.3] which determines explicit conditions on roots at a given step in the Knapp process that when satisfied show that a certain functor of cohomological induction maps the underlying module of a principal series representation to another underlying module of a principal series representation. When these conditions are satisfied at every step in the Knapp process, then one can construct a map [2, Corollary 5.6] that often allows one to conclude that Knapp's method succeeds.

The root conditions at a particular step are as follows. Let $\alpha_{j} \in \Delta^{+}\left(\mathfrak{m}_{j-1}\right)$ be the simple root chosen by the process. Form

$$
C_{j}=\left\{\gamma \in \Delta^{+}\left(\mathfrak{m}_{j-1}\right)-\left\{\alpha_{j}\right\} \mid\left\langle\gamma, \alpha_{j}\right\rangle>0,\left\langle s_{\alpha_{j}}\left(\lambda_{\sigma_{j-1}}\right), \hat{\gamma}\right\rangle \in \mathbf{Z}-\{0\}\right\} .
$$

One wants to show that if

$$
\text { if } \quad \gamma \in C_{j} \quad \text { then }\left\langle s_{\alpha_{j}}(\lambda+\delta), \hat{\gamma}\right\rangle=\left\langle\lambda+\delta, s_{\alpha_{j}}(\hat{\gamma})\right\rangle>0 \text {. }
$$

If Knapp's process ends after $n$ steps, and (2.4) holds at every step along the way, then there exists a $(\mathfrak{g}, K)$ map from a standard continuous series module to $A_{\mathfrak{q}}(\lambda)$,

$$
\Phi: X_{K}\left(\xi_{n}^{h}, v^{n}\right) \longrightarrow A_{\mathfrak{q}}(\lambda)
$$

whose image contains the $K$-type with highest weight $\Lambda$. Here, $\xi_{n}^{h}$ is a discrete series (or limit of discrete series) $\left(\mathfrak{m}_{n}, M_{n} \cap K\right)$ module with infinitesimal character $\lambda_{\sigma_{n}}$. From $\Phi$ one can often read off the Langlands parameters.

\subsection{Vogan diagrams}

A Vogan diagram is an enhancement of a Dynkin diagram from which one can clearly see whether a simple root of $\Delta^{+}(\mathfrak{g})$ is compact, noncompact or complex. In the equal rank case, this diagram is obtained from the Dynkin diagram by using hollow dots to represent the compact simple roots and black dots to represent the noncompact simple roots [5]. Using the bracket relations between $f$ and $\mathfrak{p}$, one can show that a positive root containing an even (resp. odd) number of noncompact simple roots in its decomposition into simple roots is compact (resp. noncompact). Therefore, Vogan diagrams are a wonderful way to see the structure of $\Delta_{K}$ and $\Delta(\mathfrak{p})$. Moreover, it is a simple task to determine the Vogan diagram of $\Delta_{M_{j+1}}^{+}$from that of $\Delta_{M_{j}}^{+}$throughout the Knapp process. Since we intend to get our hands dirty with explicit computations using roots to 
check Knapp's conjecture (to check conditions such as (2.4) above), we want to label a given Vogan diagram in a manner amenable to calculations.

We use the following notation to capture the information in a given Vogan diagram of classical type: $A_{n}, B_{n}, C_{n}$, or $D_{n}$. Let $\beta_{1}, \ldots, \beta_{n}$ denote the simple roots, labelled and ordered in the standard manner. Consider also, the "main $A_{n^{\prime}}$ " subdiagram; for diagrams of type $A_{n}$, let $n^{\prime}=n$; for diagrams of type $B_{n}$ and $C_{n}$, let $n^{\prime}=n-1$; for diagrams of type $D_{n}$, let $n^{\prime}=n-2$. By the "main $A_{n^{\prime}}$ " subdiagram, we mean the portion of the Vogan diagram corresponding to the simple roots $\beta_{1}, \ldots, \beta_{n^{\prime}}$. By the "tail," we mean the remaining simple roots. We use the phrase "compact tail" (resp. "noncompact tail") if all of the simple roots in the tail are compact (resp. noncompact). In the case of a $D_{n}$ diagram, a "mixed tail" means that exactly one of the two roots in the tail is noncompact. In this case, without loss of generality, we assume $\beta_{n}$ is noncompact. Moreover, in the case of a noncompact tail $D_{n}$ diagram, we may assume $\left\langle\lambda+\delta, \beta_{n-1}\right\rangle \geq\left\langle\lambda+\delta, \beta_{n}\right\rangle$.

Let $\beta_{\mathfrak{p}_{1}}, \ldots, \beta_{\mathfrak{p}_{m}}$ be an enumeration of the noncompact simple roots in the main $A_{n^{\prime}}$, with $\mathfrak{p}_{i}<\mathfrak{p}_{j}$ if $i<j$, and let $\mathfrak{p}_{0}=0$. Let

$$
s_{i}=\mathfrak{p}_{i+1}-\mathfrak{p}_{i}-1, \quad \text { and } \quad c_{i}=\sum_{j=\mathfrak{p}_{i}+1}^{\mathfrak{p}_{i+1}-1} \beta_{j} \quad \text { for } 0 \leq i \leq m-1
$$

$$
s_{m}=n^{\prime}-\mathfrak{p}_{m}, \quad \text { and } \quad c_{m}=\sum_{j=\mathfrak{p}_{m}+1}^{n^{\prime}} \beta_{j} .
$$

Loosely, $s_{i}$ is the number of compact simple roots between $\beta_{\mathfrak{p}_{i}}$ and $\beta_{\mathfrak{p}_{i+1}}$ and $c_{i}$ is the sum of these simple roots. Further, let

$$
\kappa_{i}=\beta_{\mathfrak{p}_{i}}+c_{i}+\beta_{\mathfrak{p}_{i+1}}, \quad \text { for } \quad 1 \leq i \leq m-1 .
$$

These are (some of the) simple roots of $\Delta^{+}(\mathfrak{f}, \mathrm{t})$.

Suppose the Vogan diagram is of type $B_{n}, C_{n}$, or $D_{n}$. Let $\beta_{\mathfrak{p}_{i}}$ be in the main $A_{n^{\prime}}$. Now form the Vogan subdiagram with $\beta_{\mathfrak{p}_{i}}$ as its first simple root. Let $P_{i}$ be the highest root in this subdiagram that is orthogonal to $\beta_{\mathfrak{p}_{i}}$. For example, if $\beta_{\mathfrak{p}_{i}}=e_{j}-e_{j+1}$, then $P_{i}=e_{j}+e_{j+1}$. In particular,

$$
P_{m}=\beta_{\mathfrak{p}_{m}}+c_{m}+E, \quad \text { where } E= \begin{cases}c_{m}+2 \beta_{n} & \text { for } B_{n} \\ c_{m}+\beta_{n} & \text { for } C_{n} \\ c_{m}+\beta_{n-1}+\beta_{n} & \text { for } D_{n}\end{cases}
$$




\section{Preliminary Lemmas}

To start, we would like to interpret the condition that $\Lambda$ is $K$-dominant in terms of $\lambda+\delta$. To do this, we make use of the assumption that $l$ is built from a subset of the compact simple roots, for in this case

$$
\Lambda=\lambda+2 \delta(\mathfrak{l} \cap \mathfrak{p})=\lambda+2 \delta(\mathfrak{p})=(\lambda+\delta)+\left(\delta-2 \delta_{K}\right) .
$$

Therefore, if $\kappa \in \Delta^{+}(\mathfrak{f}, \mathrm{t})$, then

$$
\langle\Lambda, \kappa\rangle \geq 0 \Leftrightarrow\langle\lambda+\delta, \kappa\rangle \geq\left\langle 2 \delta_{K}-\delta, \kappa\right\rangle .
$$

Since $2 \delta_{K}-\delta$ is a function of the Vogan diagram, one can easily describe $\Lambda$ $K$-dominance in terms of the infinitesimal character $\lambda+\delta$, the $\Delta^{+}(\mathfrak{f}, \mathrm{t})$ simple roots and the structural $s_{i}$ parameters. We summarize this information in the last column of the following table.

Lemma 3.1. In terms of the Vogan diagram $s_{i}$ 's and $c_{i}$ 's in (2.6), the $\Delta^{+}(\mathfrak{f}, \mathrm{t})$ simple roots and the condition of $\Lambda K$-dominance are as in the table below.

\begin{tabular}{|c|c|c|c|}
\hline Diagram & Case & $\Delta^{+}(\mathfrak{f}, \mathfrak{t})$ simple & $\langle\lambda+\delta, \mathfrak{f}$-simple $\rangle \geq$ \\
\hline$A_{n}-D_{n}$ & & $\begin{array}{l}\beta_{i} \text { compact simple in main } A_{n^{\prime}} \\
\kappa_{i}, 1 \leq i \leq m-1\end{array}$ & $\begin{array}{l}1 \\
-s_{i}\end{array}$ \\
\hline$A_{n}$ & & no others & \\
\hline$B_{n}$ & $\begin{array}{l}\beta_{n} \text { compact } \\
1 . s_{m-1} \neq 0 \\
2 . s_{m-1}=0 \\
\text { a. } m \leq 2 \\
\text { b. } m \geq 3\end{array}$ & $\begin{array}{l}\beta_{n} \\
\kappa=\beta_{\mathfrak{p}_{m}-1}+\beta_{\mathfrak{p}_{m}}+P_{m} \\
\text { no others } \\
\kappa=\kappa_{m-2}+\beta_{\mathfrak{p}_{m}}+P_{m}\end{array}$ & $\begin{array}{l}1 / 2 \\
-2\left(s_{m}+1\right) \\
-\left(s_{m-2}+2 s_{m}+3\right)\end{array}$ \\
\hline$B_{n}$ & $\begin{array}{l}\beta_{n} \text { noncompact } \\
\text { 1. } s_{m} \neq 0 \\
\text { 2. } s_{m}=0 \\
\text { a. } m=1 \\
\text { b. } m \geq 2\end{array}$ & $\begin{array}{l}\kappa_{m}=\beta_{\mathfrak{p}_{m}}+c_{m}+\beta_{n} \\
\kappa=\beta_{n-1}+2 \beta_{n} \\
\text { no others } \\
\kappa=\kappa_{m-1}+2 \beta_{n}\end{array}$ & $\begin{array}{l}-\left(s_{m}+1 / 2\right) \\
0\end{array}$ \\
\hline$C_{n}$ & $\beta_{n}$ compact & $\begin{array}{l}\beta_{n} \\
\kappa=\beta_{\mathfrak{p}_{m}}+P_{m}\end{array}$ & $\begin{array}{l}2 \\
-2 s_{m}\end{array}$ \\
\hline
\end{tabular}

TABLE 1. 
TABLE 1. (continued)

\begin{tabular}{clll}
\hline Diagram & Case & $\Delta^{+}(\mathfrak{f}, \mathfrak{t})$ simple & $\langle\lambda+\delta, \mathfrak{f}$-simple $\rangle \geq$ \\
\hline \multirow{2}{*}{$C_{n}$} & $\beta_{n}$ noncompact & $\kappa_{m}=\beta_{\mathfrak{p}_{m}}+c_{m}+\beta_{n}$ & $-\left(s_{m}+1\right)$ \\
& & & \\
$D_{n}$ & Tail compact & $\beta_{n-1}$ & 1 \\
& & $\beta_{n}$ & 1 \\
& 1. $s_{m-1} \neq 0$ & $\kappa=\beta_{\mathfrak{p}_{m}-1}+\beta_{\mathfrak{p}_{m}}+P_{m}$ & $-\left(2 s_{m}+3\right)$ \\
& 2. $s_{m-1}=0$ & & \\
a. $m \leq 2$ & no others & $-\left(s_{m-2}+2 s_{m}+4\right)$ \\
& b. $m \geq 3$ & $\kappa=\kappa_{m-2}+\beta_{\mathfrak{p}_{m}}+P_{m}$ & \\
& & & \\
$D_{n}$ & Tail mixed & $\beta_{n-1}$ & $-s_{m}$ \\
& & $\kappa_{m}=\beta_{\mathfrak{p}_{m}}+c_{m}+\beta_{n}$ & \\
& & & $-s_{m}$ \\
$D_{n}$ & Tail noncompact & $\kappa_{m_{a}}=\beta_{\mathfrak{p}_{m}}+c_{m}+\beta_{n-1}$ & \\
& & $\kappa_{m_{b}}=\beta_{\mathfrak{p}_{m}}+c_{m}+\beta_{n}$ & $-s_{m}$ \\
& 1. $s_{m} \neq 0$ & $\kappa=\beta_{n-2}+\beta_{n-1}+\beta_{n}$ & -1 \\
& 2. $s_{m}=0$ & & \\
a. $m=1$ & no others & \\
& b. $m \geq 2$ & $\kappa=\kappa_{m-1}+\beta_{n-1}+\beta_{n}$ & $-\left(s_{m-1}+2\right)$ \\
& & &
\end{tabular}

Consequently, if $\Lambda$ is $K$-dominant then $\left\langle\lambda+\delta, \kappa_{i}+c_{i}\right\rangle \geq-s_{i}+s_{i}=0$ for $1 \leq i \leq m-1$.

The power of the results of [2] is that the question of determining the Langlands parameters of $A_{\mathfrak{q}}(\lambda)$ is often reduced to a question about roots. In general, if $\alpha$ is the noncompact simple root selected at a particular step of the Knapp process, then we are to consider the set $C$ of positive roots at that step with a positive inner product versus $\alpha$ (excluding $\alpha$ ) and show that for all $\gamma \in C$

$$
\begin{aligned}
\text { if } \quad\left\langle s_{\alpha}(\lambda+\delta), \hat{\gamma}\right\rangle \in \mathbf{Z}-\{0\} \\
\text { then }\left\langle s_{\alpha}(\lambda+\delta), \hat{\gamma}\right\rangle=\left\langle\lambda+\delta, s_{\alpha}(\hat{\gamma})\right\rangle \geq 0 .
\end{aligned}
$$

Throughout this paper, we assume that $\Lambda$ is $K$-dominant. Let $\kappa$ be a compact simple root, so that by Lemma $3.1\langle\lambda+\delta, \kappa\rangle>0$. If $\gamma \in C$ such that $\gamma-\kappa \in C$ then $\langle\lambda+\delta, \gamma\rangle>\langle\lambda+\delta, \gamma-\kappa\rangle$. This enables us to reduce the set of roots in $C$ for which we need to check condition (3.1) to the roots in $C$ that are contained in Table 2 below. 
TABLE 2 .

\begin{tabular}{|c|c|c|c|}
\hline Case & Diagram & Roots to check & \\
\hline$\alpha=\beta_{\mathfrak{p}_{i}}$ & $A_{n}-D_{n}$ & $\gamma_{j}^{A}=\left\{\begin{array}{l}\beta_{\mathfrak{p}_{i}}+c_{i}+\cdots+\beta_{\mathfrak{p}_{j}} \\
\beta_{\mathfrak{p}_{j}}+c_{j}+\cdots+\beta_{\mathfrak{p}_{i}}\end{array}\right.$ & $\begin{array}{l}\text { for } i<j \leq m \\
\text { for } 1 \leq j<i\end{array}$ \\
\hline \multirow[t]{5}{*}{$\alpha=\beta_{\mathfrak{p}_{i}}$} & $A_{n}-D_{n}$ & $\gamma_{j}^{A}=\left\{\begin{array}{l}\beta_{\mathfrak{p}_{i}}+c_{i}+\cdots+\beta_{\mathfrak{p}_{j}} \\
\beta_{\mathfrak{p}_{j}}+c_{j}+\cdots+\beta_{\mathfrak{p}_{i}}\end{array}\right.$ & $\begin{array}{l}\text { for } i<j \leq m \\
\text { for } 1 \leq j<i\end{array}$ \\
\hline & $B_{n}-D_{n}$ & $\gamma_{j}=\left\{\begin{array}{l}\gamma_{j}^{A}+P_{j} \\
\beta_{\mathfrak{p}_{i}-1}+\beta_{\mathfrak{p}_{i}}+P_{i} \\
\gamma_{j}^{A}+P_{i}\end{array}\right.$ & $\begin{array}{l}\text { for } i<j \leq m \\
\text { for } j=i, \mathfrak{p}_{i} \neq 1 \\
\text { for } 1 \leq j<i\end{array}$ \\
\hline & $B_{n}-D_{n}$ & $\gamma^{S}=\beta_{\mathfrak{p}_{i}}+\cdots+\beta_{n}$ & \\
\hline & $B_{n}$ & $\gamma^{S+}=\gamma^{S}+\beta_{n}$ & \\
\hline & $C_{n}$ & $\gamma_{i}^{\prime}=\beta_{\mathfrak{p}_{i}}+P_{i}$ & \\
\hline$\alpha=\beta_{n}$ & $B_{n}$ & $\gamma_{j}^{A}=\beta_{\mathfrak{p}_{j}}+\cdots+\beta_{n-1}+2 \beta_{n}$ & for $1 \leq j \leq m$ \\
\hline$\alpha=\beta_{n}$ & $C_{n}$ & $\gamma_{j}^{A}=\beta_{\mathfrak{p}_{j}}+\cdots+\beta_{n}$ & for $1 \leq j \leq m$ \\
\hline \multirow[t]{2}{*}{$\alpha=\beta_{n}$} & $D_{n}$ & $\begin{array}{l}\gamma_{j}^{A}=\beta_{\mathfrak{p}_{j}}+\cdots+c_{m}+\beta_{n} \\
\gamma^{\prime}=\beta_{n-2}+\beta_{n-1}+\beta_{n}\end{array}$ & for $1 \leq j \leq m$ \\
\hline & & $\gamma_{j}^{S}=\beta_{\mathfrak{p}_{j}}+\cdots+\beta_{n}$ & for $1 \leq j \leq m$ \\
\hline
\end{tabular}

Next we make a few simple computations.

Lemma 3.2. Assume $\Lambda$ is $K$-dominant. If $\alpha=\beta_{\mathfrak{p}_{i}}$ is the noncompact simple root selected by the Knapp process, then the roots $\gamma_{j}^{A}$ satisfy

$$
\left\langle\lambda+\delta, s_{\alpha}\left(\gamma_{j}^{A}\right)\right| \geq 0 \quad \text { for } 1 \leq(j \neq i) \leq m .
$$

Moreover, in the notation of (2.6), equality exists exactly when

(1) $\left\langle\lambda+\delta, c_{x}\right\rangle=s_{x}$, for all $i \leq x<j$ if $i<j$, and all $j \leq x<i$ if $j<i$,

(2) $\left\langle\lambda+\delta, \kappa_{x}\right\rangle=-s_{x}$, for all $i \leq x<j$ if $i<j$, and all $j \leq x<i$ if $j<i$, and

(3) $\left\langle\lambda+\delta, \beta_{\mathfrak{p}_{i}}\right\rangle=\left\langle\lambda+\delta, \beta_{\mathfrak{p}_{j}}\right\rangle$. 
Proof. Without loss of generality, we may assume $i<j$. Since $\left|\beta_{\mathfrak{p}_{i}}\right|^{2}=$ $\left|\gamma_{j}^{A}\right|^{2}$ so that $s_{\alpha}\left(\gamma_{j}^{A}\right)=\gamma_{j}^{A}-\beta_{\mathfrak{p}_{i}}$, we compute

$$
\begin{aligned}
& 2\left\langle\lambda+\delta, \gamma_{j}^{A}-\beta_{\mathfrak{p}_{i}}\right\rangle=\left\langle\lambda+\delta, \sum_{x=i}^{j-1}\left(c_{x}+\kappa_{x}\right)\right\rangle+\left\langle\lambda+\delta, \beta_{\mathfrak{p}_{j}}-\beta_{\mathfrak{p}_{i}}\right\rangle \\
& \geq\left\langle\lambda+\delta, \sum_{x=i}^{j-1} c_{x}\right\rangle-\sum_{x=i}^{j-1} s_{x}+\left\langle\lambda+\delta, \beta_{\mathfrak{p}_{j}}-\beta_{\mathfrak{p}_{i}}\right\rangle \\
& \text { by Lemma } 3.1 \\
& \geq\left\langle\lambda+\delta, \beta_{\mathfrak{p}_{j}}-\beta_{\mathfrak{p}_{i}}\right\rangle \quad \text { by Lemma } 3.1 \\
& \geq 0 \quad \text { by (P1) }
\end{aligned}
$$

to obtain the result.

Lemma 3.3. Assume $\Lambda$ is $K$-dominant. If $1 \leq j \leq m-1$ then $\left\langle\lambda+\delta, P_{j}\right\rangle \geq$ $\left\langle\lambda+\delta, P_{j+1}\right\rangle$, with equality exactly when

(1) $\left\langle\lambda+\delta, c_{j}\right\rangle=s_{j}$, and

(2) $\left\langle\lambda+\delta, \kappa_{j}\right\rangle=-s_{j}$.

Proof. An application of Lemma 3.1 gives

$$
\begin{aligned}
\left\langle\lambda+\delta, P_{j}\right\rangle & =\left\langle\lambda+\delta, \kappa_{j}+c_{j}+P_{j+1}\right\rangle \\
& \geq-s_{j}+\left\langle\lambda+\delta, c_{j}\right\rangle+\left\langle\lambda+\delta, P_{j+1}\right\rangle \\
& \geq\left\langle\lambda+\delta, P_{j+1}\right\rangle
\end{aligned}
$$

for the result.

Lemma 3.4. Assume $\Lambda$ is $K$-dominant and $\left\langle\lambda+\delta, P_{m}\right\rangle \geq 0$. If $\alpha=\beta_{\mathfrak{p}_{i}}$ is the noncompact simple root selected by the Knapp process, then the roots $\gamma_{j}$ satisfy

$$
\left\langle\lambda+\delta, s_{\alpha}\left(\gamma_{j}\right)\right\rangle \geq 0 .
$$

Proof. Since $\left|\beta_{\mathfrak{p}_{i}}\right|^{2}=\left|\gamma_{j}\right|^{2}$ we have $s_{\beta_{\mathfrak{v}_{i}}}\left(\gamma_{j}\right)=\gamma_{j}-\beta_{\mathfrak{p}_{i}}$. If $j \neq i$, then

$$
\begin{array}{rlrl}
\left\langle\lambda+\delta, \gamma_{j}-\beta_{\mathfrak{p}_{i}}\right\rangle & =\left\langle\lambda+\delta, \gamma_{j}^{A}-\beta_{\mathfrak{p}_{i}}+P_{x}\right\rangle & & \text { for some } x \geq i \\
& =\left\langle\lambda+\delta, s_{\alpha}\left(\gamma_{j}^{A}\right)\right\rangle+\left\langle\lambda+\delta, P_{x}\right\rangle & \\
& \geq\left\langle\lambda+\delta, P_{m}\right\rangle \geq 0 . & \text { by Lemma } 3.3
\end{array}
$$


Next suppose that $j=i$ and $\mathfrak{p}_{i} \neq 1$. Then

$$
\left\langle\lambda+\delta, \gamma_{i}-\beta_{\mathfrak{p}_{i}}\right\rangle=\left\langle\lambda+\delta, \beta_{\mathfrak{p}_{i}-1}+P_{i}\right\rangle \geq\left\langle\lambda+\delta, \beta_{\mathfrak{p}_{i}-1}\right\rangle
$$

by Lemma 3.3. If $\beta_{\mathfrak{p}_{i}-1}$ is compact then by Lemma $3.1\left\langle\lambda+\delta, \beta_{\mathfrak{p}_{i}-1}\right\rangle \geq 1$. If $\beta_{\mathfrak{p}_{i}-1}$ is noncompact, then Lemma 3.1 implies $\left\langle\lambda+\delta, \beta_{\mathfrak{p}_{i}-1}+\beta_{\mathfrak{p}_{i}}\right\rangle \geq 0$ so that $\left\langle\lambda+\delta, \beta_{\mathfrak{p}_{i}-1}\right\rangle \geq-\left\langle\lambda+\delta, \beta_{\mathfrak{p}_{i}}\right\rangle \geq 1$ by $(\mathrm{P} 1)$.

\section{Main theorems}

As a remarkable consequence of the simple lemmas in Section 3, we obtain the following theorem.

THEOREM 4.1. Suppose the Vogan diagram is of type $A_{n}, C_{n}$ with a compact tail, or $D_{n}$ with a mixed tail. Let $\mathfrak{q}=\mathfrak{l} \oplus \mathfrak{u}$ be a $\theta$ stable parabolic subalgebra where $\{$ is formed from a subset of compact simple roots. If $\Lambda$ is $K$-dominant, then the hypotheses of Corollary 5.6 of [2] are satisfied. Therefore there exists $a(\mathfrak{g}, K)$ map from a standard continuous series module to $A_{\mathfrak{q}}(\lambda)$,

$$
\Phi: X_{K}\left(\xi_{n}^{h}, v^{n}\right) \longrightarrow A_{\mathfrak{q}}(\lambda),
$$

whose image contains the $K$-type with highest weight $\Lambda$.

Remark. Theorem 4.1 handles the Lie algebras $\mathfrak{s} \mathfrak{H}(p, q), \mathfrak{s p}(p, q)$ and $\mathfrak{S O}^{*}(2 n)$.

COROLlary 4.2. Under the hypotheses in Theorem 4.1, let $V$ be the irreducible subquotient of $A_{\mathfrak{q}}(\lambda)$ containing the $K$-type with highest weight $\Lambda$. The map (4.1), and therefore Knapp's method, gives a set of Langlands parameters of $V$ if any of the following hold:

(1) $A_{\mathfrak{q}}(\lambda)$ is irreducible,

(2) $A_{\mathfrak{q}}(\lambda)$ is infinitesimally unitary,

(3) $A_{\mathfrak{q}}(\lambda)$ is weakly fair,

(4) $\Lambda$ is the minimal $K$-type of $X_{K}(\sigma, v)$,

(5) $\Lambda$ is the minimal $K$-type of $A_{\mathfrak{q}}(\lambda)$.

Proof of Corollary 4.2. This is [2, Corollary 6.10].

Proof of Theorem 4.1. For the three Vogan diagrams in question, we show that the $K$-dominance of $\Lambda$ implies that (3.1) holds, and then that the diagram type essentially remains the same throughout the Knapp process. These two points, coupled with the fact that $\Lambda$-dominance persists throughout the process will prove the theorem. 
We start by showing that (3.1) holds for the roots listed in Table 2. Suppose $\alpha=\beta_{\mathfrak{p}_{i}}$ is the noncompact simple root of $\Delta^{+}(\mathfrak{g})$ selected by the Knapp process. For all Vogan diagrams, Lemma 3.2 handles the roots $\gamma_{j}^{A}$. For an $A_{n}$ diagram, these are the only roots that need to be checked. Therefore, (3.1) holds for an $A_{n}$ diagram.

For the roots $\gamma_{j}$ of Table 2, we show the following claim so that Lemma 3.4 provides the result.

Claim 4.3. If the Vogan diagram is of type $C_{n}$ with a compact tail (that is, $\mathfrak{S p}(p, q))$ or $D_{n}$ with a mixed tail (that is, $\left.\mathfrak{S}_{\mathfrak{D}^{*}}(2 n)\right)$, then $\left\langle\lambda+\delta, P_{m}\right\rangle \geq 1$.

Proof of Claim 4.3. For $C_{n}$, using Lemma 3.1 we compute

$$
\left\langle\lambda+\delta, P_{m}\right\rangle=\left\langle\lambda+\delta, \frac{1}{2} \kappa+c_{m}+\frac{1}{2} \beta_{n}\right\rangle \geq-s_{m}+s_{m}+1=1 .
$$

For $D_{n}$, using Lemma 3.1 we compute

$$
\left\langle\lambda+\delta, P_{m}\right\rangle=\left\langle\lambda+\delta, \kappa_{m}+c_{m}+\beta_{n-1}\right\rangle \geq\left\langle\lambda+\delta, \beta_{n-1}\right\rangle \geq 1 .
$$

This completes the proof of Claim 4.3.

Next we consider the root $\gamma^{S}$. In the $C_{n}$ case, since $\left|\beta_{\mathfrak{p}_{i}}\right|^{2}=\left|\gamma^{S}\right|^{2}$ we have $s_{\beta_{v_{i}}}\left(\gamma^{S}\right)=\gamma^{S}-\beta_{\mathfrak{p}_{i}}$. If $i=m$, then we have

$$
\left\langle\lambda+\delta, \gamma^{S}-\beta_{\mathfrak{p}_{m}}\right\rangle=\left\langle\lambda+\delta, c_{m}+\beta_{n}\right\rangle \geq s_{m}+\left\langle\lambda+\delta, \beta_{n}\right\rangle \geq 2 .
$$

Similiarly, if $i<m$, we may use Lemma 3.2 to deduce the same inequality. $\left\langle\lambda+\delta, \gamma^{S}-\beta_{\mathfrak{p}_{i}}\right\rangle=\left\langle\lambda+\delta, \gamma_{m}^{A}-\beta_{\mathfrak{p}_{i}}\right\rangle+\left\langle\lambda+\delta, c_{m}+\beta_{n}\right\rangle \geq s_{m}+\left\langle\lambda+\delta, \beta_{n}\right\rangle \geq 2$.

In the $D_{n}$ case, we write

$$
\gamma^{S}=\left(\beta_{\mathfrak{p}_{i}}+\cdots+c_{m}+\beta_{n}\right)+\left(\beta_{n-1}\right) .
$$

Since $\beta_{\mathfrak{p}_{i}}+\cdots+c_{m}+\beta_{n}$ lies in an $A_{n-1}$ subdiagram, and $\beta_{n-1}$ is a compact simple root, Lemmas 3.2 and 3.1 combine to give $\left\langle\lambda+\delta, \gamma^{S}-\alpha\right\rangle \geq 1$. This concludes the proof that (3.1) holds when $\alpha=\beta_{\mathfrak{p}_{i}}$ in the $D_{n}$ case.

Continuing the proof in the $C_{n}$ case, we are only left to consider the root $\gamma_{i}^{\prime}$. Since $\beta_{\mathfrak{p}_{i}}$ is a short root and $\gamma_{i}^{\prime}$ is a long root, we have

$$
\begin{aligned}
\left\langle\lambda+\delta, s_{\beta_{p_{i}}}\left(\gamma_{i}^{\prime}\right)\right\rangle & =\left\langle\lambda+\delta, \gamma_{i}^{\prime}-2 \beta_{\mathfrak{p}_{i}}\right\rangle & & \\
& =\left\langle\lambda+\delta, P_{i}-\beta_{\mathfrak{p}_{i}}\right\rangle & & \\
& \geq\left\langle\lambda+\delta, P_{m}\right\rangle+1 & & \text { by Lemma } 3.3 \text { and }(\mathrm{P} 2) \\
& \geq 2 & & \text { by Claim } 4.2 .
\end{aligned}
$$


This concludes the proof that (3.1) holds in the $C_{n}$ case.

We are only left to consider the $D_{n}$ case in which $\alpha=\beta_{n}$. Arguments as in Lemma 3.1 handle $\gamma_{j}^{A}$ and $\gamma^{\prime}$. Moreover, the $D_{n}$ argument above for $\gamma^{S}$ also handles the $\gamma_{j}^{S}$. This completes the proof that (3.1) holds in the $D_{n}$ case.

Claim 4.4. For the three Vogan diagrams in the theorem, the subsequent Vogan diagrams arising in the Knapp process are of the same type, possibly together with a union of singleton $A_{1}$ diagrams.

Proof OF Claim 4.4. For an $A_{n}$ diagram this is clear; if the Vogan diagram of $\Delta^{+}(\mathfrak{g})$ is $A_{m}$, then the diagram of $\Delta^{+}\left(\mathfrak{m}_{1}\right)$ is $A_{m-2}$.

Next, suppose the Vogan diagram is a $C_{n}$ with a compact tail. Let $\alpha=\beta_{\mathfrak{p}_{x}}$ be the noncompact simple root of $\Delta^{+}(\mathfrak{g})$ selected by the Knapp process. The diagram of $\Delta^{+}\left(\mathfrak{m}_{1}\right)$ is $C_{n-2} \cup A_{1}$. The $A_{1}$ node is the root $P_{x}$. Since $P_{x}$ is orthogonal but not strongly orthogonal to $\alpha$, it is compact in $\Delta^{+}\left(\mathfrak{m}_{1}\right)$. Moreover, $P_{x}$ is orthogonal to all other positive roots in $\Delta^{+}\left(\mathfrak{m}_{1}\right)$ and is therefore a compact singleton $A_{1}$ in the Vogan diagram for $\Delta^{+}\left(\mathfrak{m}_{1}\right)$. To show that the $C_{n-2}$ part of $\Delta^{+}\left(\mathfrak{m}_{1}\right)$ has a compact tail, we consider the following two cases.

(1) If $x \leq n-2$, then the tail root of the $C_{n-2}$ part is $\beta_{n} \in \Delta^{+}(\mathrm{g})$. Since $\beta_{n}$ is strongly orthogonal to $\alpha$ it remains compact as a root in $\Delta^{+}\left(\mathfrak{m}_{1}\right)$.

(2) If $x=n-1$, then the tail root of the $C_{n-2}$ part is $2 \beta_{n-2}+2 \beta_{n-1}+\beta_{n}$. This root is compact as a root in $\Delta^{+}(\mathfrak{g})$. It remains compact in $\Delta^{+}\left(\mathfrak{m}_{1}\right)$ since it is strongly orthogonal to $\beta_{n-1}$.

This completes the proof of the claim for $C_{n}$. Another simple case-by-case argument shows that subsequent diagrams are also of type $D_{n}$ with a mixed tail together with a union of noncompact $A_{1}$ singletons. This completes the proof of Claim 4.4.

Claim 4.4 and [4, Proposition 10] therefore give the result for $A_{n}$. For the $C_{n}$ case, the singleton roots are always compact and therefore not chosen in the Knapp process. In the $D_{n}$ case, Claim 4.3 implies that no noncompact singleton will be chosen during the Knapp process. This completes the proof of Theorem 4.1.

Under any of the conditions outlined in Corollary 4.2, Theorem 4.1 proves that for $S U(p, q), S p(p, q)$, and $S O^{*}(2 n)$, the conjectural method of [4] to produce Langlands parameters is correct.

Before we prove theorems similiar to Theorem 4.1 for the remaining classical diagrams, we highlight two properties of the compact tail $C_{n}$ case and the mixed $D_{n}$ case of that theorem.

(1) $\Lambda K$-dominant implies that $\left\langle\lambda+\delta, P_{m}\right\rangle \geq 0$. Therefore, since the property of $\Lambda$ being $K$-dominant is inherited throughout the process, and the type of Vogan diagram remains the same, 
(2) the property $\left\langle\lambda+\delta, P_{m}\right\rangle \geq 0$ is inherited throughout the process.

Unfortunately, for the remaining classical Vogan diagrams, neither of these properties necessarily holds.

Example 4.5. Consider a $D_{8}$ Vogan diagram with a noncompact tail. Let $m=1$ and $\mathfrak{p}_{1}=3$ so that $s_{0}=2$ and $s_{1}=3$. Suppose

$$
\left\langle\lambda+\delta, \beta_{i}\right\rangle=\left\{\begin{array}{rl}
1 & \text { for } i=1,2,5,6 \\
-6 & \text { for } i=\mathfrak{p}_{1}=3 \\
2 & \text { for } i=4 \\
-1 & \text { for } i=7,8
\end{array} .\right.
$$

One checks that $\Lambda$ is $K$-dominant and $\left\langle\lambda+\delta, P_{m}\right\rangle=0$. The Knapp process selects $\alpha=\beta_{\mathfrak{p}_{m}}$ and one shows that (3.1) holds at this first step.

Continuing the process, the Vogan diagram at the second step is a noncompact tail $D_{6} \cup A_{1}$ with $D_{6}$ data $m=1$ and $\mathfrak{p}_{1}=2$ so that $s_{0}=1, s_{1}=2$. At this step, we have

$$
\left\langle\lambda+\delta, \beta_{i}\right\rangle=\left\{\begin{aligned}
1 & \text { for } i=1,3,4 \\
-3 & \text { for } i=\mathfrak{p}_{1}=2 \\
-1 & \text { for } i=5,6
\end{aligned}\right.
$$

Although the appropriate $\Lambda$ is $K$-dominant, we now have $\left\langle\lambda+\delta, P_{m}\right\rangle=-1$. The Knapp process selects $\alpha=\beta_{\mathfrak{p}_{m}}$ and one shows that (3.1) still holds.

At the next step, the Vogan diagram is a noncompact $D_{4} \cup A_{1} \cup A_{1}$ with $D_{4}$ data $m=1$ and $\mathfrak{p}_{1}=1$ so that $s_{0}=0$ and $s_{1}=1$. We now have

$$
\left\langle\lambda+\delta, \beta_{i}\right\rangle=\left\{\begin{array}{rl}
1 & \text { for } i=2 \\
-1 & \text { for } i=1,3,4
\end{array} .\right.
$$

Again, $\left\langle\lambda+\delta, P_{m}\right\rangle=-1$. If the Knapp process selects any of the noncompact simple roots in the $D_{4}$ piece, say $\beta_{1}$, then (3.1) fails with respect to the sum of the simple roots, $\gamma^{S} \in C$ :

$$
\left\langle\lambda+\delta, s_{\beta_{1}}\left(\gamma^{S}\right)\right\rangle=\left\langle\lambda+\delta, \beta_{2}+\beta_{3}+\beta_{4}\right\rangle=1-1-1=-1 .
$$

As we see from Example 4.5, $\Lambda$ being $K$-dominant may not be a sufficient condition to ensure that (3.1) holds. In fact, there are simple one-step examples that show this is the case for the five classical Vogan diagrams not considered in Theorem 4.1, not just the noncompact tail $D_{n}$ case of Example 4.5. Therefore, the techniques of [2] are insufficient to settle Knapp's conjecture. 
However, for the classical Vogan diagrams not considered in Theorem 4.1, we can show the following series of theorems. Theorem 4.6 assumes one condition in addition to $\Lambda$ being $K$-dominant so that (3.1) holds at a single particular stage in the Knapp process. Theorem 4.7 assumes a slightly stronger condition so that (3.1) holds at every stage in the Knapp process. Theorem 4.9 explores what happens assuming that $\Lambda$ is $K$-dominant and that $A_{\mathfrak{q}}(\lambda)$ is in the weakly fair range. These modules are of particular interest since they are underlying modules of the Flensted-Jensen discrete series. Since the proofs are computational, we present them in the sections following the statements of the theorems.

THEOREM 4.6. Let $\alpha$ be the noncompact simple root chosen by the Knapp process. Suppose the classical Vogan diagram is NOT one of the following two types

(1) $C_{n}$ with

(a) $\beta_{n}$ noncompact,

(b) $\alpha \neq \beta_{n}$, and

(c) $\left\langle\lambda+\delta, \beta_{n}\right\rangle<-s_{m}$, or

(2) $D_{n}$ with

(a) noncompact tail,

(b) $s_{m}=1$, and

(c) $\left\langle\lambda+\delta, \beta_{n-1}\right\rangle=\left\langle\lambda+\delta, \beta_{n}\right\rangle \leq-2$,

(d) $\left\langle\lambda+\delta, \kappa_{m}\right\rangle=\left\langle\lambda+\delta, \kappa_{m_{a}}\right\rangle=\left\langle\lambda+\delta, \kappa_{m_{b}}\right\rangle=-1$

If $\Lambda$ is $K$-dominant and $\left\langle\lambda+\delta, P_{m}\right\rangle \geq 0$, then $\langle\lambda+\delta, \gamma\rangle>0$ for all $\gamma \in C$.

Remark. As Example 4.5 shows, the property $\left\langle\lambda+\delta, P_{m}\right\rangle \geq 0$ may not be inherited throughout the Knapp process. If it is inherited in a particular example, and the subsequent Vogan diagrams are not one of the above two types, then repeated application of Theorem 4.6 yields the same conclusion as in Theorem 4.1. (We can also see from Example 4.5 that the property $\langle\lambda+$ $\left.\delta, P_{m}\right\rangle \geq 0$ is not a necessary condition for (3.1) to hold at a particular step.)

Ideally, one would like to find a property that when coupled with $\Lambda K$ dominance implies (3.1) and is inherited by the subsequent diagrams arising in the process. If we sacrifice just a little bit regarding $\left\langle\lambda+\delta, P_{m}\right\rangle$ we can find one.

THEOREM 4.7. Suppose the classical Vogan diagram is not of the following type

(1) $C_{n}$ with 
(a) $\beta_{n}$ noncompact,

(b) $\alpha \neq \beta_{n}$, and

(c) $\left\langle\lambda+\delta, \beta_{n}\right\rangle<-s_{m}$

Suppose further that $\Lambda$ is $K$-dominant and $\left\langle\lambda+\delta, P_{m}\right\rangle \geq\left\langle\lambda+\delta, c_{m}\right\rangle-s_{m}$. Then the hypotheses of Corollary 5.6 of [2] are satisfied. Therefore there exists $a(\mathfrak{g}, K)$ map from a standard continuous series module to $A_{\mathfrak{q}}(\lambda)$,

$$
\Phi: X_{K}\left(\xi_{n}^{h}, v^{n}\right) \longrightarrow A_{\mathfrak{q}}(\lambda),
$$

whose image contains the $K$-type with highest weight $\Lambda$.

REMark. This successfully handles the $S O(m, n)$ cases. The excluded $S p(n, \mathrm{R})$ case remains, at this time, an unresolved thorn.

COROLlary 4.8. If the Vogan diagram has a compact tail, $\Lambda$ is $K$-dominant, and

$$
\left\langle\lambda+\delta, \beta_{\mathfrak{p}_{m}}\right\rangle \geq\left\{\begin{array}{ll}
-2 s_{m}-1 & \text { for } B_{n} \\
-2 s_{m}-2 & \text { for } D_{n}
\end{array},\right.
$$

then Theorem 4.7 applies.

Proof. Under condition (4.2), Lemma 3.1 implies $\left\langle\lambda+\delta, P_{m}\right\rangle \geq\langle\lambda+$ $\left.\delta, c_{m}\right\rangle-s_{m}$.

TheOREm 4.9. Suppose $\Lambda$ is $K$-dominant, and that $A_{\mathfrak{q}}(\lambda)$ is in the weakly fair range. Then $\langle\lambda+\delta, \gamma\rangle \geq 0$ for all $\gamma \in C$. That is, the first stage of the Knapp process holds.

REMARK 1. Unfortunately, the property of a Vogan diagram being weakly fair is not necessarily inherited throughout the Knapp process. (This can be seen by modifying Example 4.5 by extending the $D_{8}$ diagram to a $D_{18}$ diagram by changing $s_{0}$ from 2 to 12 , setting $\left\langle\lambda+\delta, \beta_{j}\right\rangle=1$ for the "new" compact simple roots $\left\{\beta_{j}\right\}_{j=1}^{10}$, and forming $\mathfrak{l}$ from all compact simple roots with inner product $=1$ versus $\lambda+\delta$.) But, of course, if the subsequent Vogan diagrams are all weakly fair, then repeated application of this theorem yields the same conclusion as in Theorem 4.1.

REMARK 2. Example 4.5, in its original guise, also shows that the weakly fair condition is not necessary for (3.1) to hold at a particular step.

REMARK 3. The weakly fair $A_{\mathfrak{q}}(\lambda)$ 's (without requiring that $\mathfrak{l} \subseteq \mathfrak{f}$ ) such that $\Lambda=\lambda+2 \delta(\mathfrak{t} \cap \mathfrak{p})$ is $K$-dominant are the underlying modules of the FlenstedJensen discrete series [1]. In [9], Schlichtkrull found the Langlands parameters of most of these modules. In fact, Corollary 11.229 of [6] is essentially an $A_{\mathfrak{q}}(\lambda)$ 
formulation of this result. In [10], under an additional assumption - loosely, that the restriction of $\lambda+\delta$ to the compact part of a maximally split Cartan subalgebra of $\mathfrak{I}$ satisfies a weakly fair condition - Schlichtkrull reduces the question of Langlands parameters for the Flensted-Jensen modules to the case in which $\mathfrak{l} \subseteq f$. The four theorems of this section then handle most of these.

\section{Proofs and other results}

Before coming to the proofs, let us restate and label our basic assumptions. We always assume that $\operatorname{rank} G=\operatorname{rank} K$ and the following:

(AS0) $\mathfrak{I}$ is formed from a subset of compact simple roots;

(AS1) If $\alpha$ is the noncompact simple root chosen by the Knapp process and $\beta$ is another noncompact simple root, then

$$
\begin{aligned}
& \langle\lambda+\delta, \hat{\beta}\rangle \geq\langle\lambda+\delta, \hat{\alpha}\rangle \\
& \langle\lambda+\delta, \hat{\alpha}\rangle \in \mathbf{Z}_{\leq-1}
\end{aligned}
$$

(AS2) $\Lambda$ is $K$-dominant, so that Lemma 3.1 holds.

In general, we want to show that for the roots $\gamma$ listed in Table 2

$$
\begin{aligned}
\text { if } \quad\left\langle s_{\alpha}(\lambda+\delta), \hat{\gamma}\right\rangle \in \mathrm{Z}-\{0\} \\
\text { then } \quad\left\langle s_{\alpha}(\lambda+\delta), \hat{\gamma}\right\rangle=\left\langle\lambda+\delta, s_{\alpha}(\hat{\gamma})\right\rangle \geq 0
\end{aligned}
$$

Under (AS0)-(AS2), Theorem 4.1 gives the best result for diagrams of type $A_{n}, C_{n}$ with a compact tail, and $D_{n}$ with a mixed tail. In the proofs of the other theorems, we only consider the remaining classical Vogan diagrams.

\subsection{Proof of Theorem 4.6}

In Theorem 4.6, we make the following additional assumption

$$
\left\langle\lambda+\delta, P_{m}\right\rangle \geq 0
$$

We start the proof with diagrams of type $B_{n}$. First consider the case $\alpha=$ $\beta_{n}$, so that the tail is necessarily noncompact. In this case, (P1) implies that $\left\langle\lambda+\delta, \beta_{\mathfrak{p}_{j}}\right\rangle \geq\left\langle\lambda+\delta, 2 \beta_{n}\right\rangle$ for $1 \leq j \leq m$. According to Table 2 , we only 
need to check (5.1) for the roots $\gamma_{j}^{A}$. We compute

$$
\begin{array}{rlrl}
\left\langle\lambda+\delta, s_{\alpha}\left(\gamma_{j}^{A}\right)\right\rangle & =\left\langle\lambda+\delta, \gamma_{j}^{A}-2 \beta_{n}\right\rangle & \\
& =\frac{1}{2}\left\langle\lambda+\delta, \beta_{\mathfrak{p}_{j}}+\sum_{x=j}^{m-1}\left(\kappa_{x}+c_{x}\right)+\beta_{\mathfrak{p}_{m}}+2 c_{m}\right\rangle \\
& \geq \frac{1}{2}\left\langle\lambda+\delta, \beta_{\mathfrak{p}_{j}}+\beta_{\mathfrak{p}_{m}}+2 c_{m}\right\rangle & & \text { by Lemma } 3.1 \\
& \geq \frac{1}{2}\left\langle\lambda+\delta, \beta_{\mathfrak{p}_{m}}+2 c_{m}+2 \beta_{n}\right. & & \text { by (P1) } \\
& =\left\langle\lambda+\delta, P_{m}\right\rangle & \\
& \geq 0 & & \text { by (AS3). }
\end{array}
$$

Therefore, (5.1) holds for $B_{n}$ diagrams when $\alpha=\beta_{n}$.

Now consider the case where $\alpha=\beta_{\mathfrak{p}_{i}}$. The roots $\gamma_{j}^{A}$ and $\gamma_{j}$ of Table 2 are handled by Lemmas 3.2 and 3.4; we are only left to consider $\gamma^{S}$ and $\gamma^{S+}$. Since $\beta_{\mathfrak{p}_{i}}$ is a long root, $s_{\beta_{\mathfrak{p}_{i}}}\left(\gamma^{S}\right)=\gamma^{S}-\beta_{\mathfrak{p}_{i}}$ and $s_{\beta_{\mathfrak{p}_{i}}}\left(\gamma^{S+}\right)=\gamma^{S+}-\beta_{\mathfrak{p}_{i}}$.

First suppose that $\left\langle\lambda+\delta, \beta_{n}\right\rangle \geq 0$. If $i=m$ then

$$
\left\langle\lambda+\delta, \gamma^{S+}-\beta_{\mathfrak{p}_{i}}\right\rangle \geq\left\langle\lambda+\delta, \gamma^{S}-\beta_{\mathfrak{p}_{i}}\right\rangle=\left\langle\lambda+\delta, c_{m}+\beta_{n}\right\rangle \geq s_{m} \geq 0 .
$$

If $i<m$, then

$$
\begin{aligned}
\left\langle\lambda+\delta, \gamma^{S+}-\beta_{\mathfrak{p}_{i}}\right\rangle & \geq\left\langle\lambda+\delta, \gamma^{S}-\beta_{\mathfrak{p}_{i}}\right\rangle \\
& =\left\langle\lambda+\delta, \gamma_{m}^{A}-\beta_{\mathfrak{p}_{i}}\right\rangle+\left\langle\lambda+\delta, c_{m}+\beta_{n}\right\rangle \geq s_{m} \geq 0 .
\end{aligned}
$$

Next suppose that $\left\langle\lambda+\delta, \beta_{n}\right\rangle \leq-\frac{1}{2}$. Then $\beta_{n}$ is noncompact so that (P1) implies $\left\langle\lambda+\delta, 2 \beta_{n}\right\rangle \geq\left\langle\lambda+\delta, \beta_{\mathfrak{p}_{i}}\right\rangle$. Therefore

$$
\begin{array}{rlr}
\left\langle\lambda+\delta, \gamma^{S}-\beta_{\mathfrak{p}_{i}}\right\rangle>\left\langle\lambda+\delta, \gamma^{S+}-\beta_{\mathfrak{p}_{i}}\right\rangle & \\
& =\frac{1}{2}\left\langle\lambda+\delta,-\beta_{\mathfrak{p}_{i}}+\sum_{x=i}^{m-1}\left(\kappa_{x}+c_{x}\right)+\beta_{\mathfrak{p}_{m}}+2 c_{m}+4 \beta_{n}\right\rangle \\
& \geq \frac{1}{2}\left\langle\lambda+\delta,-\beta_{\mathfrak{p}_{i}}+\beta_{\mathfrak{p}_{m}}+2 c_{m}+4 \beta_{n}\right\rangle & \text { by Lemma } 3.1 \\
& \geq \frac{1}{2}\left\langle\lambda+\delta, \beta_{\mathfrak{p}_{m}}+2 c_{m}+2 \beta_{n}\right\rangle & \\
& =\frac{1}{2}\left\langle\lambda+\delta, P_{m}\right\rangle & \\
\geq 0 & \text { by (P1) } \\
&
\end{array}
$$


This completes the case where $\alpha=\beta_{\mathfrak{p}_{i}}$, and therefore Vogan diagrams of type $B_{n}$.

Suppose now that the diagram is of type $C_{n}$ with a noncompact tail. First consider the case where $\alpha=\beta_{n}$. In this case, (P1) gives $\left\langle\lambda+\delta, \beta_{\mathfrak{p}_{j}}\right\rangle \geq$ $\frac{1}{2}\left\langle\lambda+\delta, \beta_{n}\right\rangle$ so that $\left\langle\lambda+\delta, \beta_{\mathfrak{p}_{j}}-\beta_{n}\right\rangle \geq-\frac{1}{2}\left\langle\lambda+\delta, \beta_{n}\right\rangle$. We then compute

$$
\begin{aligned}
& \left\langle\lambda+\delta, s_{\beta_{n}}\left(\gamma_{j}^{A}\right)\right\rangle=\left\langle\lambda+\delta, \gamma_{j}^{A}-\beta_{n}\right\rangle \\
& =\frac{1}{2}\left\langle\lambda+\delta, \sum_{x=j}^{m} \kappa_{x}+c_{x}\right\rangle+\frac{1}{2}\left\langle\lambda+\delta, \beta_{\mathfrak{p}_{j}}-\beta_{n}\right\rangle \\
& \geq \frac{1}{2}\left[-\left(s_{m}+1\right)+s_{m}\right]-\frac{1}{2}\left\langle\lambda+\delta, \beta_{n}\right\rangle \text { by Lemma 3.1, (P1) } \\
& \geq-\frac{1}{2}+1>0,
\end{aligned}
$$

for the result in this case.

Now consider the case where $\alpha=\beta_{\mathfrak{p}_{i}}$. The roots $\gamma_{j}^{A}$ and $\gamma_{j}$ are handled by Lemmas 3.2 and 3.4; we are only left to consider the roots $\gamma_{i}^{\prime}$ and $\gamma^{S}$.

Since $\beta_{\mathfrak{p}_{i}}$ is a short root and $\gamma_{i}^{\prime}$ is a long root, we have

$$
\begin{aligned}
\left\langle\lambda+\delta, s_{\beta_{\mathfrak{p}_{i}}}\left(\gamma_{i}^{\prime}\right)\right\rangle & =\left\langle\lambda+\delta, \gamma_{i}^{\prime}-2 \beta_{\mathfrak{p}_{i}}\right\rangle & & \\
& =\left\langle\lambda+\delta, P_{i}-\beta_{\mathfrak{p}_{i}}\right\rangle & & \\
& \geq\left\langle\lambda+\delta, P_{m}\right\rangle+1 & & \text { by Lemma } 3.3 \text { and (P2) } \\
& \geq 1 & & \text { by (AS3). }
\end{aligned}
$$

The root $\gamma^{S}$ is the one that gives us difficulties and forces us to exclude the $C_{n}$ diagram in the statement of the theorem. Since $\left|\beta_{\mathfrak{p}_{i}}\right|^{2}=\left|\gamma^{S}\right|^{2}$ we have $s_{\beta_{\mathfrak{p}_{i}}}\left(\gamma^{S}\right)=\gamma^{S}-\beta_{\mathfrak{p}_{i}}$. If $i=m$, then we have

$$
\left\langle\lambda+\delta, \gamma^{S}-\beta_{\mathfrak{p}_{m}}\right\rangle=\left\langle\lambda+\delta, c_{m}+\beta_{n}\right\rangle \geq s_{m}+\left\langle\lambda+\delta, \beta_{n}\right\rangle .
$$

Similiarly, if $i<m$, we may use Lemma 3.2 to deduce the same inequality.

$$
\left\langle\lambda+\delta, \gamma^{S}-\beta_{\mathfrak{p}_{i}}\right\rangle=\left\langle\lambda+\delta, \gamma_{m}^{A}-\beta_{\mathfrak{p}_{i}}\right\rangle+\left\langle\lambda+\delta, c_{m}+\beta_{n}\right\rangle \geq s_{m}+\left\langle\lambda+\delta, \beta_{n}\right\rangle .
$$

Since $\beta_{n}$ is noncompact (recall that Theorem 4.1 handles the compact tail $C_{n}$ diagrams), the additional hypothesis $\left\langle\lambda+\delta, \beta_{n}\right\rangle \geq-s_{m}$ then implies the result. This completes the case where $\alpha=\beta_{\mathfrak{p}_{i}}$, and therefore the allowed Vogan diagrams of type $C_{n}$.

Suppose now that the diagram is of type $D_{n}$ and consider the case where $\alpha=\beta_{n}$. Since $\alpha$ is a tail root, we are dealing with a noncompact tail. The roots 
$\gamma_{j}^{A}$ and $\gamma^{\prime}$ are roots in an $A_{n}$-type subdiagram and therefore arguments as in Lemma 3.2 handle them. We are left with only the $\gamma_{j}^{S}$ to consider. Since we are in a single line diagram, $s_{\beta_{n}}\left(\gamma_{j}^{S}\right)=\gamma_{j}^{S}-\beta_{n}$. Additionally, we may assume that $m \geq 1$ and $n \geq 4$.

First suppose $s_{m}=0$. For $j=m$ we have that

$$
\left\langle\lambda+\delta, \gamma_{m}^{S}-\beta_{n}\right\rangle=\left\langle\lambda+\delta, \kappa_{m_{a}}\right\rangle \geq-s_{m}=0, \quad \text { by Lemma 3.1. }
$$

For $j \leq m-1$, we have

$$
\begin{aligned}
&\left\langle\lambda+\delta, \gamma_{j}^{S}-\beta_{n}\right\rangle=\langle\lambda+\left.\delta,\left(\frac{1}{2} \sum_{x=j}^{m-2} k_{x}+c_{x}\right)\right\rangle \\
&+\left\langle\lambda+\delta, \frac{1}{2} \beta_{\mathfrak{p}_{j}}+\frac{1}{2} \beta_{\mathfrak{p}_{m-1}}+c_{m-1}+\beta_{\mathfrak{p}_{m}}+\beta_{n-1}\right\rangle \\
& \geq\left\langle\lambda+\delta, \frac{1}{2} \beta_{\mathfrak{p}_{j}}+\frac{1}{2} \beta_{\mathfrak{p}_{m-1}}+c_{m-1}+\beta_{\mathfrak{p}_{m}}+\beta_{n-1}\right\rangle \text { by Lemma 3.1 } \\
& \geq\left\langle\lambda+\delta, c_{m-1}+\beta_{\mathfrak{p}_{m}}+\beta_{n-1}+\beta_{n}\right\rangle \text { by (P1) } \\
&=\left\langle\lambda+\delta, c_{m-1}+P_{m}\right\rangle \quad \text { by Lemma 3.1, (AS3). } \\
& \geq s_{m-1} \geq 0 \quad
\end{aligned}
$$

This settles the $s_{m}=0$ case.

Next suppose $s_{m} \geq 2$. Similiarly we compute

$$
\begin{aligned}
\left\langle\lambda+\delta, \gamma_{j}^{S}-\beta_{n}\right\rangle=\langle\lambda+ & \left.\delta,\left(\frac{1}{2} \sum_{x=j}^{m-1} k_{x}+c_{x}\right)\right\rangle \\
& +\left\langle\lambda+\delta, \frac{1}{2} \beta_{\mathfrak{p}_{j}}+\frac{1}{2} \beta_{\mathfrak{p}_{m}}+c_{m}+\beta_{n-1}\right\rangle
\end{aligned}
$$

$$
\begin{aligned}
& \geq\left\langle\lambda+\delta, \frac{1}{2} \beta_{\mathfrak{p}_{j}}+\frac{1}{2} \beta_{\mathfrak{p}_{m}}+c_{m}+\beta_{n-1}\right\rangle \quad \text { by Lemma } 3.1 \\
& \geq\left\langle\lambda+\delta, c_{m}+\beta_{n-1}+\beta_{n}\right\rangle \quad \text { by }(\mathrm{P} 1) \\
& =\left\langle\lambda+\delta,\left(c_{m}-\beta_{n-2}\right)+\kappa\right\rangle \quad \text { since } s_{m} \geq 1 \\
& \geq\left(s_{m}-1\right)-1 \geq 0 \quad \text { by Lemma } 3.1 .
\end{aligned}
$$

This settles the $s_{m}=2$ case.

Finally, suppose $s_{m}=1$. If $\langle\lambda+\delta, \kappa\rangle \neq-1$, then integrality and Lemma 3.1 imply that $\langle\lambda+\delta, \kappa\rangle \geq 0$. Continuing from (5.5) above we then get 
that $\left\langle\lambda+\delta, \gamma_{j}^{S}-\beta_{n}\right\rangle \geq\langle\lambda+\delta, \kappa\rangle \geq 0$. If $\langle\lambda+\delta, \kappa\rangle=-1$ and either $\left\langle\lambda+\delta, \beta_{n-1}\right\rangle>\left\langle\lambda+\delta, \beta_{n}\right\rangle$ (strictly) or $\left\langle\lambda+\delta, \kappa_{m_{b}}\right\rangle \neq-1$ then we continue from (5.3) above so that

$$
\begin{aligned}
\left\langle\lambda+\delta, \gamma_{j}^{S}-\beta_{n}\right\rangle & \geq\left\langle\lambda+\delta, \frac{1}{2} \beta_{\mathfrak{p}_{j}}+\frac{1}{2} \beta_{\mathfrak{p}_{m}}+c_{m}+\beta_{n-1}\right\rangle \\
& \geq\left\langle\lambda+\delta, \frac{1}{2} \beta_{\mathfrak{p}_{m}}+\beta_{n-2}+\beta_{n-1}+\frac{1}{2} \beta_{n}\right\rangle \\
& =\frac{1}{2}\left(\left\langle\lambda+\delta, \kappa_{m_{a}}\right\rangle+\langle\lambda+\delta, \kappa\rangle\right) \\
& =\frac{1}{2}\left(\left\langle\lambda+\delta, \kappa_{m_{b}}\right\rangle+\left\langle\lambda+\delta, \beta_{n-1}-\beta_{n}\right\rangle-1\right) \\
& >-1 .
\end{aligned}
$$

Integrality then implies that $\left\langle\lambda+\delta, \gamma_{j}^{S}-\beta_{n}\right\rangle \geq 0$.

Therefore, we have shown that if $\alpha=\beta_{n}$ then we have the result except if $s_{m}=1$ (so that $c_{m}=\beta_{n-2}$ and $\beta_{\mathfrak{p}_{m}}=\beta_{n-3}$ ) and

$$
\begin{aligned}
&\left\langle\lambda+\delta, \beta_{n-3}\right\rangle=\left\langle\lambda+\delta, \beta_{n-1}\right\rangle=\left\langle\lambda+\delta, \beta_{n}\right\rangle=-x, \\
& \text { and } \quad\left\langle\lambda+\delta, \beta_{n-2}\right\rangle=2 x-1 .
\end{aligned}
$$

Then condition (AS3) and integrality imply $x \geq 2$. Since this is precisely the $D_{n}$ situation excluded in the theorem, this completes the case where $\alpha=\beta_{n}$.

Now consider the case where $\alpha=\beta_{\mathfrak{p}_{i}}$. The roots $\gamma_{j}^{A}$ and $\gamma_{j}$ are handled by Lemmas 3.2 and 3.4; we are only left to consider $\gamma^{S}$; we are to show that $\left\langle\lambda+\delta, \gamma^{S}-\beta_{\mathfrak{p}_{i}}\right\rangle \geq 0$.

First suppose the tail is compact. If $i=m$, then $\gamma^{S}-\beta_{\mathfrak{p}_{i}}$ is a sum of compact simple roots and Lemma 3.1 gives the result. If $i<m$, then $\gamma^{S}=$ $\gamma_{m}^{A}+c_{m}+\beta_{n-1}+\beta_{n}$. Since $c_{m}+\beta_{n-1}+\beta_{n}$ is a sum of compact simple roots orthogonal to $\beta_{\mathfrak{p}_{i}}$, Lemmas 3.1 and 3.2 give the result.

Now suppose the tail is noncompact. If $s_{m}=0$ and $i=m$ then $\gamma^{S}$ lies in an $A_{n}$ type subdiagram and Lemma 3.2 gives the result. If $i=m-1$, then

$$
\begin{aligned}
\left\langle\lambda+\delta, \gamma^{S}-\beta_{\mathfrak{p}_{m-1}}\right\rangle & =\left\langle\lambda+\delta, c_{m-1}+\beta_{\mathfrak{p}_{m}}+\beta_{n-1}+\beta_{n}\right\rangle \\
& =\left\langle\lambda+\delta, c_{m-1}+P_{m}\right\rangle \geq s_{m-1},
\end{aligned}
$$


by Lemma 3.1 and (AS3). If $i \leq m-2$, then similiarly,

$$
\begin{aligned}
\left\langle\lambda+\delta, \gamma^{S}-\beta_{\mathfrak{p}_{i}}\right\rangle= & \left\langle\lambda+\delta, \gamma_{m-1}^{A}-\beta_{\mathfrak{p}_{i}}\right\rangle \\
& +\left\langle\lambda+\delta, c_{m-1}+\beta_{\mathfrak{p}_{m}}+\beta_{n-1}+\beta_{n}\right\rangle \\
\geq & \left\langle\lambda+\delta, c_{m-1}+\beta_{\mathfrak{p}_{m}}+\beta_{n-1}+\beta_{n}\right\rangle \quad \text { by Lemma } 3.2 \\
\geq & s_{m-1} \quad \text { by Lemma 3.1 and (AS3). }
\end{aligned}
$$

This handles the case when $s_{m}=0$.

If $s_{m} \geq 2$ and $i=m$ then $\gamma^{S}-\beta_{\mathfrak{p}_{m}}=c_{m}+\beta_{n-1}+\beta_{n}$ and then computations from (5.5) onward give the result. Similiarly, if $i \leq m-1$, then $\gamma^{S}-\beta_{\mathfrak{p}_{i}}=$ $\left(\gamma_{m}^{A}-\beta_{\mathfrak{p}_{i}}\right)+c_{m}+\beta_{n-1}+\beta_{n}$ and Lemma 3.1 along with the computations from (5.4) onward give the result.

So we are left again with the case of $s_{m}=1$. Clearly, by the symmetry of a $D_{4}$ diagram, if $i=m$ then we have the result except in the cases excluded in the $s_{m}=1, \alpha=\beta_{n}$ case above. If $i<m$ then

$$
\left\langle\lambda+\delta, \gamma^{S}-\beta_{\mathfrak{p}_{i}}\right\rangle=\left\langle\lambda+\delta, \gamma_{m}^{A}-\beta_{\mathfrak{p}_{i}}\right\rangle+\langle\lambda+\delta, \kappa\rangle \quad \text { by Lemma 3.1. }
$$

The first term is $\geq 0$ by Lemma 3.2, while the second term is $\geq-1$ by Lemma 3.1. Hence by integrality, if either inequality is strict, then $\left\langle\lambda+\delta, \gamma^{S}-\right.$ $\left.\beta_{\mathfrak{p}_{i}}\right\rangle \geq 0$. Lemma 3.2 characterizes exactly when this occurs. In particular, if $\left\langle\lambda+\delta, \gamma_{m}^{A}-\beta_{\mathfrak{p}_{i}}\right\rangle=0$, then $\left\langle\lambda+\delta, \beta_{\mathfrak{p}_{i}}\right\rangle=\left\langle\lambda+\delta, \beta_{\mathfrak{p}_{m}}\right\rangle$ so that we could have chosen $\alpha=\beta_{\mathfrak{p}_{m}}$ and we have already discussed this case. This completes the $\alpha=\beta_{\mathfrak{p}_{i}}$ case in $D_{n}$, and therefore completes the proof of the theorem.

\subsection{Proof of Theorem 4.7}

In this theorem, we assume (AS0)-(AS2) as well as

$$
\left\langle\lambda+\delta, P_{m}\right\rangle \geq\left\langle\lambda+\delta, c_{m}\right\rangle-s_{m} .
$$

Since conditions (AS3') and (AS2) imply (AS3) of Theorem 4.6, the results of that theorem apply. Further, hypothesis (AS3') excludes the noncompact tail $D_{n}$ case we needed to avoid in Theorem 4.6. Therefore, we are only left with showing that hypothesis $\left(\mathrm{AS}^{\prime}\right)$ is inherited throughout the Knapp process.

To do this, we need to understand how a Vogan diagram changes at the different stages of the Knapp process. This is easy, but notationally a bit cumbersome. We use $V^{x}$ to denote the Vogan diagram of $\Delta^{+}\left(\mathfrak{m}_{x}, \mathrm{t}^{x}\right)$, and we denote the corresponding $\beta_{\mathfrak{p}_{i}}, \kappa_{i}, \alpha, c_{i}, s_{i}$, and $P_{i}$ with a superscript: $\beta_{\mathfrak{p}_{i}}^{x}, \kappa_{i}^{x}$, $\alpha^{x}, c_{i}^{x}, s_{i}^{x}$, and $P_{i}^{x}$. Further, if $\alpha^{x}=\beta_{\mathfrak{p}_{i}}^{x}$ is the noncompact simple root of $V^{x}$ chosen by the Knapp process then define

$$
\beta_{\alpha}^{x}=\beta_{\mathfrak{p}_{i}-1}^{x}+\beta_{\mathfrak{p}_{i}}^{x}+\beta_{\mathfrak{p}_{i}+1}^{x} .
$$


We start by listing what happens to the Dynkin diagram in passing from $V^{x}$ to $V^{x+1}$. If $\alpha^{x}$ is a singleton in $V^{x}$, then $V^{x+1}=V^{x} \backslash\left\{\alpha^{x}\right\}$. Otherwise

$$
\begin{aligned}
A_{n} \longmapsto A_{n-2}, & B_{n} \longmapsto\left\{\begin{array}{ll}
B_{n-2} \cup A_{1}, & \alpha^{x} \in \text { main } A_{n^{\prime}} \\
B_{n-1}, & \alpha=\beta_{n}
\end{array},\right. \\
D_{n} \longmapsto D_{n-2} \cup A_{1}, & C_{n} \longmapsto\left\{\begin{array}{ll}
C_{n-2} \cup A_{1}, & \alpha^{x} \in \text { main } A_{n^{\prime}} \\
C_{n-1}, & \alpha=\beta_{n}
\end{array} .\right.
\end{aligned}
$$

(Recall that the cases of $A_{n}, C_{n}$ with a compact tail, and $D_{n}$ with a mixed tail are already handled by Theorem 4.1.) The additional $A_{1}$ component in $V^{x+1}$ is precisely $P_{i}^{x}$ when $\alpha=\beta_{\mathfrak{p}_{i}}$. Assumption (AS3) ensures that this root will never be selected by the Knapp process. Anyhow, since $P_{i}^{x}$ is orthogonal all other positive roots of $V^{x+1}$, it plays no role when we need to check condition (5.1).

Our primary concern lies with how $P_{m}^{x}, s_{m}^{x}$, and $c_{m}^{x}$ are affected by the Knapp process. We summarize this data in Table 3.

\begin{tabular}{|c|c|c|c|}
\hline Case & $P_{m}^{x+1}$ & $s_{m}^{x+1}$ & $c_{m}^{x+1}$ \\
\hline I. $\quad \alpha^{x}=\beta_{\mathfrak{p}_{m}-j}^{x}$ for $j \geq 2$ & $P_{m}^{x}$ & $s_{m}^{x}$ & $c_{m}^{x}$ \\
\hline \multicolumn{4}{|l|}{ II. $\quad \alpha^{x}=\beta_{\mathfrak{p}_{m}-1}^{x}$} \\
\hline A. $s_{m-2}^{x}=0$ & $P_{m}^{x}+\kappa_{m-2}^{x}$ & $s_{m}^{x}$ & $c_{m}^{x}$ \\
\hline B. $s_{m-2}^{x} \neq 0$ & $P_{m-2}^{x}$ & $s_{m-2}^{x}+s_{m}^{x}$ & $\left(c_{m-2}^{x}-\beta_{\mathfrak{p}_{m-1}-1}^{x}\right)+\beta_{\alpha}^{x}+c_{m}^{x}$ \\
\hline
\end{tabular}

TABle 3.

III. $\alpha^{x}=\beta_{\mathfrak{p}_{m}}^{x}$
A. $s_{m}^{x}=0$
1. $s_{m-1}^{x}=0$
2. $s_{m-1}^{x} \neq 0$
$P_{m-2}^{x}$
$P_{m-1}^{x}$
$\begin{array}{ll}s_{m-2}^{x} & c_{m-2}^{x} \\ s_{m-1}^{x}-1 & c_{m-1}^{x}-\beta_{\mathfrak{p}_{m}-1}^{x}\end{array}$
B. $s_{m}^{x} \neq 0$
1. $s_{m-1}^{x}=0$
$\begin{array}{lll}P_{m-2}^{x} & s_{m-2}^{x}+s_{m}^{x} & c_{m-2}^{x}+\beta_{\alpha}^{x}+\left(c_{m}^{x}-\beta_{\mathfrak{p}_{m}+1}^{x}\right) \\ \beta_{\mathfrak{p}_{m}-1}^{x}+P_{m}^{x}-\beta_{\mathfrak{p}_{m}+1}^{x} & s_{m}^{x}-1 & c_{m}^{x}-\beta_{\mathfrak{p}_{m}+1}^{x}\end{array}$

IV. $\alpha^{x}=\beta_{n}^{x}$ in $B_{n}$ or $C_{n}$
A. $s_{m}^{x}=0$
$P_{m-1}^{x}$
$s_{m-1}^{x} \quad c_{m-1}^{x}$
B. $s_{m}^{x} \neq 0$
$P_{m}^{x}$
$s_{m}^{x}-1 \quad c_{m}^{x}-\beta_{n-1}^{x}$

V. $\alpha^{x}=\beta_{n}^{x}$ in $D_{n}$
A. $s_{m}^{x}=0$
1. $s_{m-1}^{x}=0$
2. $s_{m-1}^{x} \neq 0$
$P_{m-2}^{x}$
$P_{m-1}^{x}$
B. $s_{m}^{x}=1$
C. $s_{m}^{x} \geq 2$
$P_{m-1}^{x}$
$P_{m}^{x}$
$\begin{array}{ll}s_{m-2}^{x} & c_{m-2}^{x} \\ s_{m-1}^{x}-1 & c_{m-1}^{x}-\beta_{n-3}^{x}\end{array}$
$s_{m-1}^{x} \quad c_{m-1}^{x}$
$s_{m}^{x}-2 \quad c_{m}^{x}-\beta_{n-2}^{x}-\beta_{n-3}^{x}$ 
The heart of Theorem 4.7 is the following lemma:

LEMma 5.1. If the original Vogan diagram satisfies (AS3'), that is

$$
\left\langle\lambda+\delta, P_{m}\right\rangle \geq\left\langle\lambda+\delta, c_{m}\right\rangle-s_{m},
$$

then so do all subsequent diagrams arising in the Knapp process.

Proof. We proceed by induction on the stage number in the Knapp process, the base step assumed to be true. We assume that $\left\langle\lambda+\delta, P_{m}^{x}\right\rangle \geq\left\langle\lambda+\delta, c_{m}^{x}\right\rangle-s_{m}^{x}$. The proof proceeds in a case-by-case fashion, following Table 3 .

Case I follows immediately from the inductive hypothesis. Case II.A follows from the inductive hypothesis and Lemma 3.1, since $\left\langle\lambda+\delta, \kappa_{m-2}^{x}\right\rangle \geq-s_{m-2}^{x}=$ 0. For Case II.B, we compute

$$
\begin{aligned}
\langle\lambda+ & \left.\delta, P_{m}^{x+1}\right\rangle \\
& =\left\langle\lambda+\delta, P_{m-2}^{x}\right\rangle \\
& =\left\langle\lambda+\delta, c_{m-2}^{x}+\kappa_{m-2}^{x}\right\rangle+\left\langle\lambda+\delta, \kappa_{m-1}^{x}+P_{m}^{x}\right\rangle \\
& \geq\left\langle\lambda+\delta, c_{m-2}^{x}\right\rangle-s_{m-2}+\left\langle\lambda+\delta, \kappa_{m-1}^{x}+P_{m}^{x}\right\rangle \quad \text { by Lemma 3.1 } \\
& =\left\langle\lambda+\delta, c_{m-2}^{x}-\beta_{\mathfrak{p}_{m-1}-1}^{x}\right\rangle-s_{m-2}^{x}+\left\langle\lambda+\delta, \beta_{\mathfrak{p}_{m-1}-1}^{x}+\kappa_{m-1}^{x}+P_{m}^{x}\right\rangle \\
& =\left\langle\lambda+\delta, c_{m-2}^{x}-\beta_{\mathfrak{p}_{m-1}-1}^{x}\right\rangle-s_{m-2}^{x}+\left\langle\lambda+\delta, \beta_{\alpha}^{x}+P_{m}^{x}\right\rangle \\
& \geq\left\langle\lambda+\delta, c_{m-2}^{x}-\beta_{\mathfrak{p}_{m-1}-1}^{x}\right\rangle-s_{m-2}^{x}+\left\langle\lambda+\delta, \beta_{\alpha}^{x}+c_{m}^{x}\right\rangle-s_{m}^{x} \\
& =\left\langle\lambda+\delta, c_{m}^{x+1}\right\rangle-s_{m}^{x+1}
\end{aligned}
$$

for the desired inequality.

In case III.A.1, we compute

$$
\begin{aligned}
\langle\lambda+ & \left.\delta, P_{m}^{x+1}\right\rangle \\
& =\left\langle\lambda+\delta, P_{m-2}^{x}\right\rangle=\left\langle\lambda+\delta, \kappa_{m-2}^{x}+c_{m-2}^{x}+\kappa_{m-1}^{x}+P_{m}^{x}\right\rangle \\
& =\left\langle\lambda+\delta, 2 c_{m-2}^{x}\right\rangle+\left\langle\lambda+\delta, \beta_{\mathfrak{p}_{m-2}}^{x}+\beta_{\mathfrak{p}_{m-1}}^{x}+\kappa_{m-1}^{x}+P_{m}^{x}\right\rangle \\
& \geq\left\langle\lambda+\delta, 2 c_{m-2}^{x}\right\rangle+\left\langle\lambda+\delta, \beta_{\mathfrak{p}_{m}}^{x}+\beta_{\mathfrak{p}_{m-1}}^{x}+\kappa_{m-1}^{x}+P_{m}^{x}\right\rangle, \quad \text { by (P1) } \\
& \geq\left\langle\lambda+\delta, 2 c_{m-2}^{x}\right\rangle+\left\langle\lambda+\delta, 2 \kappa_{m-1}^{x}\right\rangle \quad \text { by inductive hypothesis } \\
& \geq\left\langle\lambda+\delta, c_{m-2}^{x}\right\rangle \\
& \geq\left\langle\lambda+\delta, c_{m-2}^{x}\right\rangle-s_{m-2}^{x} \\
& =\left\langle\lambda+\delta, c_{m}^{x+1}\right\rangle-s_{m}^{x+1}
\end{aligned}
$$


for the desired inequality.

In case III.A.2, we compute

$$
\begin{aligned}
\left\langle\lambda+\delta, P_{m}^{x+1}\right\rangle & =\left\langle\lambda+\delta, P_{m-1}^{x}\right\rangle=\left\langle\lambda+\delta, \kappa_{m-1}^{x}+c_{m-1}^{x}+P_{m}^{x}\right\rangle \\
& \geq\left\langle\lambda+\delta, \kappa_{m-1}^{x}+c_{m-1}^{x}\right\rangle \quad \text { by inductive hypothesis } \\
& \geq\left\langle\lambda+\delta, c_{m-1}^{x}\right\rangle-s_{m-1}^{x} \quad \text { by Lemma 3.1 } \\
& =\left\langle\lambda+\delta, c_{m-1}^{x}-\beta_{\mathfrak{p}_{m}-1}^{x}\right\rangle-\left(s_{m-1}^{x}-1\right)+\left\langle\lambda+\delta, \beta_{\mathfrak{p}_{m}-1}^{x}\right\rangle-1 \\
& \geq\left\langle\lambda+\delta, c_{m-1}^{x}-\beta_{\mathfrak{p}_{m}-1}^{x}\right\rangle-\left(s_{m-1}^{x}-1\right) \quad \text { by Lemma 3.1 } \\
& =\left\langle\lambda+\delta, c_{m}^{x+1}\right\rangle-s_{m}^{x+1}
\end{aligned}
$$

for the desired inequality.

In case III.B.1, we start by using (P1) to obtain

$$
\begin{aligned}
\left\langle\lambda+\delta, \kappa_{m-2}^{x}\right\rangle & =\left\langle\lambda+\delta, \beta_{\mathfrak{p}_{m-2}}^{x}+c_{m-2}^{x}+\beta_{\mathfrak{p}_{m-1}}^{x}\right\rangle \\
& \geq\left\langle\lambda+\delta, c_{m-2}^{x}+\beta_{\mathfrak{p}_{m-1}}^{x}+\beta_{\mathfrak{p}_{m}}^{x}\right\rangle \\
& =\left\langle\lambda+\delta, c_{m-2}^{x}+\kappa_{m-1}^{x}\right\rangle
\end{aligned}
$$

Then, since $\beta_{\alpha}^{x}-\beta_{\mathfrak{p}_{m}+1}^{x}=\beta_{\mathfrak{p}_{m}-1}^{x}+\beta_{\mathfrak{p}_{m}}^{x}=\kappa_{m-1}^{x}$, we may rewrite $c_{m}^{x+1}$ as $c_{m-2}^{x}+c_{m}^{x}+\kappa_{m-1}^{x}$. Finally, compute

$$
\begin{aligned}
\left\langle\lambda+\delta, P_{m}^{x+1}\right\rangle & =\left\langle\lambda+\delta, P_{m-2}^{x}\right\rangle=\left\langle\lambda+\delta, \kappa_{m-2}^{x}+c_{m-2}^{x}+\kappa_{m-1}^{x}+P_{m}^{x}\right\rangle \\
& \geq\left\langle\lambda+\delta, 2 c_{m-2}^{x}+2 \kappa_{m-1}^{x}+P_{m}^{x}\right\rangle \quad \text { by }(5.7) \\
& \geq\left\langle\lambda+\delta, c_{m-2}^{x}+\kappa_{m-1}^{x}+P_{m}^{x}\right\rangle \quad \text { by Lemma } 3.1 \\
& \geq\left\langle\lambda+\delta, c_{m-2}^{x}+c_{m}^{x}+\kappa_{m-1}^{x}\right\rangle-s_{m}^{x} \quad \text { by inductive hypothesis } \\
& >\left\langle\lambda+\delta, c_{m-2}^{x}+c_{m}^{x}+\kappa_{m-1}^{x}\right\rangle-s_{m-2}^{x}-s_{m}^{x} \\
& =\left\langle\lambda+\delta, c_{m}^{x+1}\right\rangle-s_{m}^{x+1}
\end{aligned}
$$

for the desired inequality. 
In case III.B.2, we compute

$$
\begin{aligned}
\langle\lambda+ & \left.\delta, P_{m}^{x+1}\right\rangle \\
& =\left\langle\lambda+\delta, \beta_{\mathfrak{p}_{m}-1}^{x}+P_{m}^{x}-\beta_{\mathfrak{p}_{m}+1}^{x}\right\rangle \\
& \geq\left\langle\lambda+\delta, \beta_{\mathfrak{p}_{m}-1}^{x}+c_{m}^{x}-\beta_{\mathfrak{p}_{m}+1}^{x}\right\rangle-s_{m}^{x} \quad \text { by inductive hypothesis } \\
& =\left\langle\lambda+\delta, \beta_{\mathfrak{p}_{m}-1}^{x}\right\rangle+\left\langle\lambda+\delta, c_{m+1}^{x}\right\rangle-s_{m}^{x} \\
& \geq 1+\left\langle\lambda+\delta, c_{m+1}^{x}\right\rangle-s_{m}^{x} \quad \text { by Lemma } 3.1 \text { since } \beta_{\mathfrak{p}_{m}-1}^{x} \text { is compact } \\
& =\left\langle\lambda+\delta, c_{m+1}^{x}\right\rangle-\left(s_{m}^{x}-1\right) \\
& =\left\langle\lambda+\delta, c_{m}^{x+1}\right\rangle-s_{m}^{x+1}
\end{aligned}
$$

for the desired inequality.

In case IV.A, we compute

$$
\begin{array}{rlrl}
\left\langle\lambda+\delta, P_{m}^{x+1}\right\rangle & =\left\langle\lambda+\delta, P_{m-1}^{x}\right\rangle=\left\langle\lambda+\delta, \kappa_{m-1}^{x}+c_{m-1}^{x}+P_{m}^{x}\right\rangle \\
& \geq\left\langle\lambda+\delta, c_{m-1}^{x}+P_{m}^{x}\right\rangle-s_{m-1}^{x} & & \text { by Lemma 3.1 } \\
& \geq\left\langle\lambda+\delta, c_{m-1}^{x}\right\rangle-s_{m-1}^{x} & & \text { by inductive hypothesis } \\
& =\left\langle\lambda+\delta, c_{m}^{x+1}\right\rangle-s_{m}^{x+1} &
\end{array}
$$

for the desired inequality.

In case IV.B, we compute

$$
\begin{aligned}
\left\langle\lambda+\delta, P_{m}^{x+1}\right\rangle & =\left\langle\lambda+\delta, P_{m}^{x}\right\rangle \\
& \geq\left\langle\lambda+\delta, c_{m}^{x}\right\rangle-s_{m}^{x} \quad \text { by inductive hypothesis } \\
& \geq\left\langle\lambda+\delta, c_{m}^{x}-\beta_{n-1}^{x}\right\rangle-\left(s_{m}^{x}-1\right) \\
& =\left\langle\lambda+\delta, c_{m}^{x+1}\right\rangle-s_{m}^{x+1} \text { by Lemma } 3.1 \text { since } \beta_{n-1}^{x} \text { is compact }
\end{aligned}
$$

for the desired inequality.

In case V.A.1, we compute

$$
\begin{array}{rlrl}
\left\langle\lambda+\delta, P_{m}^{x+1}\right\rangle & =\left\langle\lambda+\delta, P_{m-2}^{x}\right\rangle=\left\langle\lambda+\delta, \kappa_{m-2}^{x}+c_{m-2}^{x}+\kappa_{m-1}^{x}+P_{m}^{x}\right\rangle \\
& \geq\left\langle\lambda+\delta, c_{m-2}^{x}+P_{m}^{x}\right\rangle-s_{m-2}^{x} & & \text { by Lemma 3.1 } \\
& \geq\left\langle\lambda+\delta, c_{m-2}^{x}\right\rangle-s_{m-2}^{x} & & \text { by inductive hypothesis } \\
& =\left\langle\lambda+\delta, c_{m}^{x+1}\right\rangle-s_{m}^{x+1} &
\end{array}
$$


for the desired inequality.

In case V.A.2, we compute

$$
\begin{aligned}
\left\langle\lambda+\delta, P_{m}^{x+1}\right\rangle & =\left\langle\lambda+\delta, P_{m-1}^{x}\right\rangle=\left\langle\lambda+\delta, \kappa_{m-1}^{x}+c_{m-1}^{x}+P_{m}^{x}\right\rangle \\
& \geq\left\langle\lambda+\delta, c_{m-1}^{x}+P_{m}^{x}\right\rangle-s_{m-1}^{x} \quad \text { by Lemma } 3.1 \\
& \geq\left\langle\lambda+\delta, c_{m-1}^{x}\right\rangle-s_{m-1}^{x} \quad \text { by inductive hypothesis } \\
& \geq\left\langle\lambda+\delta, c_{m-1}^{x}-\beta_{n-3}^{x}\right\rangle-\left(s_{m-1}^{x}-1\right)
\end{aligned}
$$

by Lemma 3.1 since $\beta_{n-3}^{x}$ is compact

$$
=\left\langle\lambda+\delta, c_{m}^{x+1}\right\rangle-s_{m}^{x+1}
$$

for the desired inequality.

In case V.B, we compute

$$
\begin{array}{rlrl}
\left\langle\lambda+\delta, P_{m}^{x+1}\right\rangle & =\left\langle\lambda+\delta, P_{m-1}^{x}\right\rangle=\left\langle\lambda+\delta, \kappa_{m-1}^{x}+c_{m-1}^{x}+P_{m}^{x}\right\rangle \\
& \geq\left\langle\lambda+\delta, c_{m-1}^{x}+P_{m}^{x}\right\rangle-s_{m-1}^{x} & & \text { by Lemma } 3.1 \\
& \geq\left\langle\lambda+\delta, c_{m-1}^{x}\right\rangle-s_{m-1}^{x} & & \text { by inductive hypothesis } \\
& =\left\langle\lambda+\delta, c_{m}^{x+1}\right\rangle-s_{m}^{x+1} &
\end{array}
$$

for the desired inequality.

In case V.C, we compute

$$
\begin{aligned}
\left\langle\lambda+\delta, P_{m}^{x+1}\right\rangle & =\left\langle\lambda+\delta, P_{m}^{x}\right\rangle \\
& \geq\left\langle\lambda+\delta, c_{m}^{x}\right\rangle-s_{m}^{x} \\
& \geq\left\langle\lambda+\delta, c_{m}^{x}-\beta_{n-2}-\beta_{n-3}\right\rangle-\left(s_{m}^{x}-2\right)
\end{aligned}
$$

by Lemma 3.1 since $\beta_{n-2}^{x}$ and $\beta_{n-3}^{x}$ are compact

$$
=\left\langle\lambda+\delta, c_{m}^{x+1}\right\rangle-s_{m}^{x+1}
$$

for the desired inequality.

This completes the proof of Lemma 5.1 which in turn completes the proof of Theorem 4.7.

\subsection{Proof of Theorem 4.9}

In this theorem, we assume (AS0)-(AS2) as well as

$$
\langle\lambda+\delta(\mathfrak{u}), \alpha\rangle \geq 0 \quad \text { for all } \quad \alpha \in \Delta(\mathfrak{u})
$$

We start by forming $\mathfrak{I}_{\max }$ from ALL of the compact simple roots, regardless of their inner product with $\lambda$. Also, $\Delta^{+}\left(\mathfrak{I}_{\max }\right)$ is to be inherited from $\Delta^{+}(\mathfrak{g})$. 
Let $\beta$ be a noncompact simple root. For any $\alpha \in \Delta^{+}(\mathfrak{l})$, we know $\langle\alpha, \beta\rangle \leq 0$. Therefore, since $\Delta^{+}(\mathfrak{l}) \subseteq \Delta^{+}\left(\mathfrak{l}_{\max }\right)$, we have

$$
\langle\delta(\mathfrak{l}), \beta\rangle \geq\left\langle\delta\left(\mathfrak{I}_{\max }\right), \beta\right\rangle .
$$

The weakly fair assumption then implies

$$
\begin{aligned}
\langle\lambda+\delta, \beta\rangle & =\langle\lambda+\delta(\mathfrak{l}), \beta\rangle+\langle\delta(\mathfrak{l}), \beta\rangle & & \\
& \geq\langle\delta(\mathfrak{l}), \beta\rangle & & \text { by (AS4) } \\
& \geq\left\langle\delta\left(\mathfrak{I}_{\max }\right), \beta\right\rangle & & \text { by (5.8). }
\end{aligned}
$$

Since $\delta\left(\mathfrak{l}_{\max }\right)$ depends solely on the structure of the Vogan diagram, (5.10) allows us to compute an explicit lower bound on $\langle\lambda+\delta, \beta\rangle$ in terms of the structure constants $s_{i}$. In particular, we have the following lemma.

LeMma 5.2. Under the assumptions outlined above, if the Vogan diagram has a noncompact tail then

$$
\begin{gathered}
\left\langle\lambda+\delta, \beta_{\mathfrak{p}_{i}}\right\rangle \geq-\frac{1}{2}\left(s_{i-1}+s_{i}\right) \quad \text { for } 1 \leq i \leq m, \text { and } \\
\left\langle\lambda+\delta, \beta_{n}\right\rangle \geq \begin{cases}-\frac{1}{2} s_{m} & \text { for } a B_{n} \text { or } D_{n} \text { diagram } \\
-s_{m} & \text { for } a C_{n} \text { diagram }\end{cases} \\
\left\langle\lambda+\delta, \beta_{n-1}\right\rangle \geq-\frac{1}{2} s_{m} \quad \text { for a } D_{n} \text { diagram }
\end{gathered}
$$

If the Vogan diagram has a compact tail, then

$$
\begin{aligned}
& \left\langle\lambda+\delta, \beta_{\mathfrak{p}_{i}}\right\rangle \geq-\frac{1}{2}\left(s_{i-1}+s_{i}\right) \quad \text { for } 1 \leq i \leq m-1, \text { and } \\
& \left\langle\lambda+\delta, \beta_{\mathfrak{p}_{m}}\right\rangle \geq\left\{\begin{array}{l}
-\frac{1}{2} s_{m-1}-\left(s_{m}+\frac{1}{2}\right) \\
1 \\
-\frac{1}{2} s_{m-1}-\left(s_{m}+1\right) \quad \text { for } a B_{n} \text { diagram } a C_{n} \text { or } D_{n} \text { diagram }
\end{array}\right.
\end{aligned}
$$

REMARK 1 . The estimates above come about from $\delta\left(I_{\max }\right)$. Certainly more specific knowledge about $\{$ could lead to better estimates than those presented in the lemma.

Remark 2. The exceptional cases of Theorem 4.7 are excluded by (AS4) as a result of Lemma 5.2. 
Recall that $P_{m}=\beta_{\mathfrak{p}_{m}}+c_{m}+E$, where

$$
E= \begin{cases}c_{m}+2 \beta_{n} & \text { for } B_{n} \\ c_{m}+\beta_{n} & \text { for } C_{n} \\ c_{m}+\beta_{n-1}+\beta_{n} & \text { for } D_{n}\end{cases}
$$

As an immediate consequence of Lemmas 3.1 and 5.2, we have

Lemma 5.3. Suppose $\Lambda$ is $K$-dominant and $A_{\mathfrak{q}}(\lambda)$ is weakly fair. If the Vogan diagram has a noncompact tail then

$$
\begin{gathered}
\left\langle\lambda+\delta, c_{i-1}+2 \beta_{\mathfrak{p}_{i}}+c_{i}\right\rangle \geq 0 \text { for } 1 \leq i \leq m, \text { and } \\
\langle\lambda+\delta, E\rangle \geq 0 .
\end{gathered}
$$

If the Vogan diagram has a compact tail, then

$$
\begin{aligned}
&\left\langle\lambda+\delta, c_{i-1}+2 \beta_{\mathfrak{p}_{i}}+c_{i}\right\rangle \geq 0 \quad \text { for } 1 \leq i \leq m-1, \\
&\left\langle\lambda+\delta, c_{m-1}+2 \beta_{\mathfrak{p}_{m}}+c_{m}\right\rangle \geq\left\{\begin{array}{l}
-\left(s_{m}+1\right) \text { for } B_{n}, \\
-\left(s_{m}+2\right) \text { for } C_{n} \text { or } D_{n}
\end{array}\right. \text { and } \\
&\langle\lambda+\delta, E\rangle \geq\left\{\begin{array}{l}
s_{m}+1 \text { for } B_{n}, \\
s_{m}+2 \text { for } C_{n} \text { or } D_{n}
\end{array}\right.
\end{aligned}
$$

Before starting the proof of Theorem 4.9, we present some situations in which the hypotheses of either Theorem 4.6 or 4.7 are satisfied so that we obtain the conclusion of Theorem 4.9.

Proposition 5.4. Suppose $\Lambda$ is $K$-dominant and $A_{\mathfrak{q}}(\lambda)$ is weakly fair. If

$$
\beta_{\mathfrak{p}_{m}-1} \notin \Delta(\mathfrak{l})
$$

then $\left\langle\lambda+\delta, P_{m}\right\rangle \geq\left\langle\lambda+\delta, c_{m}\right\rangle-s_{m}$ so that Theorem 4.7 holds.

Proof. The weakly fair assumption implies that $\left\langle\lambda+\delta, \beta_{\mathfrak{p}_{m}}\right\rangle \geq\left\langle\delta(\mathfrak{l}), \beta_{\mathfrak{p}_{m}}\right\rangle$. Since $\beta_{\mathfrak{p}_{m}-1} \notin \Delta(\mathfrak{l})$, we get no contribution to $\left\langle\delta(\mathfrak{l}), \beta_{\mathfrak{p}_{m}}\right\rangle$ from any simple roots $\beta_{j}$ of $\Delta^{+}(\mathfrak{l})$ with $j \leq \mathfrak{p}_{m}$. Therefore we may take $s_{m-1}=0$ in (5.10) and (5.11). Consequently, for a diagram with a noncompact tail,

$$
\begin{aligned}
\left\langle\lambda+\delta, P_{m}\right\rangle & =\left\langle\lambda+\delta, \beta_{\mathfrak{p}_{m}}+c_{m}+E\right\rangle \\
& \geq\left\langle\lambda+\delta, c_{m}\right\rangle-\frac{1}{2} s_{m} \\
& \geq\left\langle\lambda+\delta, c_{m}\right\rangle-s_{m} .
\end{aligned}
$$


For a diagram with a compact tail,

$$
\begin{aligned}
\left\langle\lambda+\delta, P_{m}\right\rangle & =\left\langle\lambda+\delta, \beta_{\mathfrak{p}_{m}}+c_{m}+E\right\rangle \\
& \geq\left\langle\lambda+\delta, c_{m}\right\rangle \quad \text { by Lemmas 5.2, 5.3 } \\
& \geq\left\langle\lambda+\delta, c_{m}\right\rangle-s_{m} .
\end{aligned}
$$

This proves the proposition.

Due to Proposition 5.4, we may also assume (AS5)

$$
\beta_{\mathfrak{p}_{m}-1} \in \Delta(\mathfrak{l}) \text { so that } \beta_{\mathfrak{p}_{m}-1} \text { is compact and has }\left\langle\lambda+\delta, \beta_{\mathfrak{p}_{m}-1}\right\rangle=1 \text {. }
$$

The weakly fair assumption offers no improvement on Corollary 4.8 in the case of a compact tail diagram. In a noncompact tail diagram, we get the following result.

Proposition 5.5. Suppose $\Lambda$ is $K$-dominant, $A_{\mathfrak{q}}(\lambda)$ is weakly fair, and the Vogan diagram has a noncompact tail. If

$$
\left\langle\lambda+\delta, \beta_{\mathfrak{p}_{m}}\right\rangle \geq-s_{m}
$$

then

$$
\left\langle\lambda+\delta, P_{m}\right\rangle \geq\left\langle\lambda+\delta, c_{m}\right\rangle-s_{m}
$$

and Theorem 4.7 applies. In particular, if $s_{m} \geq s_{m-1}$ then (5.14) holds.

Proof. Since $P_{m}=\beta_{\mathfrak{p}_{m}}+c_{m}+E$, (5.12) and (5.14) combine to give (5.15). When $s_{m} \geq s_{m-1}$, Lemma 5.2 implies

$$
\left\langle\lambda+\delta, \beta_{\mathfrak{p}_{m}}\right\rangle \geq-\frac{1}{2}\left(s_{m-1}+s_{m}\right) \geq-s_{m} .
$$

This proves the proposition.

As a result of Proposition 5.5, Corollary 4.8 and integrality, we may also assume

$$
\left\langle\lambda+\delta, \beta_{\mathfrak{p}_{m}}\right\rangle \leq-s_{m}-1 \leq-1 .
$$

Proposition 5.6. If $\Lambda$ is $K$-dominant and $A_{\mathfrak{q}}(\lambda)$ is weakly fair, then

$$
\left\langle\lambda+\delta, P_{m}\right\rangle \geq-1 .
$$

Moreover, equality holds (5.16) only in the following cases: 
TABLE 4.

\begin{tabular}{cll}
\hline Diagram & Noncompact tail & Compact tail \\
\hline$B_{n}$ & $\left\langle\lambda+\delta, c_{m}\right\rangle=s_{m}$ & $\left\langle\lambda+\delta, c_{m}\right\rangle=s_{m}$ \\
& $\left\langle\lambda+\delta, \kappa_{m}\right\rangle=-\left(s_{m}+\frac{1}{2}\right)$ & $\langle\lambda+\delta, \kappa\rangle=-2\left(s_{m}+1\right)$ \\
& $\left\langle\lambda+\delta, \beta_{n}\right\rangle=-\frac{1}{2}$ & $\left\langle\lambda+\delta, \beta_{n}\right\rangle=\frac{1}{2}$ \\
& $\left\langle\lambda+\delta, \beta_{\mathfrak{p}_{m}}\right\rangle=-2 s_{m}$ & $\left\langle\lambda+\delta, \beta_{\mathfrak{p}_{m}}\right\rangle=-2\left(s_{m}+1\right)$ \\
$C_{n}$ & $\left\langle\lambda+\delta, c_{m}\right\rangle=s_{m}$ & \\
& $\left\langle\lambda+\delta, \kappa_{m}\right\rangle=-\left(s_{m}+1\right)$ & \\
& $\left\langle\lambda+\delta, \beta_{\mathfrak{p}_{m}}+\beta_{n}\right\rangle=-\left(2 s_{m}+1\right)$ & \\
$D_{n}$ & $\left\langle\lambda+\delta, c_{m}\right\rangle=s_{m}$ & $\left\langle\lambda+\delta, c_{m}\right\rangle=s_{m}$ \\
& $\left\langle\lambda+\delta, \kappa_{m_{a}}\right\rangle=\left\langle\lambda+\delta, \kappa_{m_{b}}\right\rangle=-s_{m}$ & $\langle\lambda+\delta, \kappa\rangle=-\left(2 s_{m}+3\right)$ \\
& $\left\langle\lambda+\delta, \beta_{n-1}\right\rangle=\left\langle\lambda+\delta, \beta_{n}\right\rangle=-1$ & $\left\langle\lambda+\delta, \beta_{n-1}\right\rangle=\left\langle\lambda+\delta, \beta_{n}\right\rangle=1$ \\
& $\left\langle\lambda+\delta, \beta_{\mathfrak{p}_{m}}\right\rangle=-2 s_{m}+1$ & $\left\langle\lambda+\delta, \beta_{\mathfrak{p}_{m}}\right\rangle=-\left(2 s_{m}+3\right)$ \\
\hline
\end{tabular}

Proof. We start the proof of Proposition 5.6 by considering diagrams of type $B_{n}$. First suppose that $\beta_{n}$ is noncompact. If $\beta_{n-1} \in \Delta(\mathfrak{l})$, then $\beta_{n-1}$ is compact, $\left\langle\lambda+\delta, \beta_{n-1}\right\rangle=1$, and $\langle\lambda+\delta, \kappa\rangle \geq 0$ by Lemma 3.1. This implies that $\left\langle\lambda+\delta, \beta_{n}\right\rangle \geq-\frac{1}{2}$. If $\beta_{n-1} \notin \Delta(\mathfrak{l})$, then the weakly fair assumption forces $\left\langle\lambda+\delta, \beta_{n}\right\rangle \geq 0$. Therefore, in either case, we have $\left\langle\lambda+\delta, \beta_{n}\right\rangle \geq-\frac{1}{2}$. Now compute

$$
\begin{aligned}
\left\langle\lambda+\delta, P_{m}\right\rangle & =\left\langle\lambda+\delta, \beta_{\mathfrak{p}_{m}}+2 c_{m}+2 \beta_{n}\right\rangle \\
& =\left\langle\lambda+\delta, \kappa_{m}+c_{m}+\beta_{n}\right\rangle \\
& \geq-\left(s_{m}+\frac{1}{2}\right)+s_{m}+\left\langle\lambda+\delta, \beta_{n}\right\rangle \quad \text { by Lemma } 3.1 \\
& =\left\langle\lambda+\delta, \beta_{n}\right\rangle-\frac{1}{2} \\
& \geq-1
\end{aligned}
$$

with equality if and only if $\left\langle\lambda+\delta, \beta_{n}\right\rangle=-\frac{1}{2},\left\langle\lambda+\delta, c_{m}\right\rangle=s_{m}$ and $\left\langle\lambda+\delta, \kappa_{m}\right\rangle=$ $-\left(s_{m}+\frac{1}{2}\right)$.

Next consider the case where $\beta_{n}$ is compact. We then compute

$$
\begin{aligned}
\left\langle\lambda+\delta, P_{m}\right\rangle & =\left\langle\lambda+\delta, \beta_{\mathfrak{p}_{m}}+2 c_{m}+2 \beta_{n}\right\rangle \\
& =\left\langle\lambda+\delta, \frac{1}{2}\left(\kappa-\beta_{\mathfrak{p}_{m}-1}\right)+c_{m}+\beta_{n}\right\rangle \quad \text { by (AS5) } \\
& \geq \frac{1}{2}\left[-2\left(s_{m}+1\right)-1\right]+s_{m}+\frac{1}{2} \quad \text { by Lemmas } 3.1 \text { and } 5.2 \\
& =-1
\end{aligned}
$$

with equality if and only if $\left\langle\lambda+\delta, c_{m}\right\rangle=s_{m},\left\langle\lambda+\delta, \beta_{n}\right\rangle=\frac{1}{2}$, and $\langle\lambda+\delta, \kappa\rangle=$ $-2\left(s_{m}+1\right)$. 
We continue by considering diagrams of type $C_{n}$ with $\beta_{n}$ noncompact. We then have

$$
\begin{aligned}
\left\langle\lambda+\delta, P_{m}\right\rangle & =\left\langle\lambda+\delta, \kappa_{m}+c_{m}\right\rangle \\
& \geq-\left(s_{m}+1\right)+s_{m} \quad \text { by Lemma } 3.1 \\
& =-1
\end{aligned}
$$

with equality if and only if $\left\langle\lambda+\delta, c_{m}\right\rangle=s_{m}$ and $\left\langle\lambda+\delta, \kappa_{m}\right\rangle=-\left(s_{m}+1\right)$.

Next we consider diagrams of type $D_{n}$. First suppose the tail is noncompact. If $\left\langle\lambda+\delta, \beta_{n}\right\rangle \geq 0$, then Lemma 3.1 implies

$$
\left\langle\lambda+\delta, P_{m}\right\rangle=\left\langle\lambda+\delta, \kappa_{m_{a}}+c_{m}\right\rangle+\left\langle\lambda+\delta, \beta_{n}\right\rangle \geq\left\langle\lambda+\delta, \beta_{n}\right\rangle \geq 0 .
$$

If $\left\langle\lambda+\delta, \beta_{n}\right\rangle \leq-1$. Then Lemma 5.2 implies $s_{m} \geq 2$, so that we can compute

$$
\begin{aligned}
\left\langle\lambda+\delta, P_{m}\right\rangle & =\left\langle\lambda+\delta, \kappa_{m_{b}}-\beta_{n}\right\rangle+\left\langle\lambda+\delta,\left(c_{m}-\beta_{n-2}\right)\right\rangle+\langle\lambda+\delta, \kappa\rangle \\
& \geq\left(-s_{m}+1\right)+\left(s_{m}-1\right)-1 \quad \text { by Lemma } 3.1 \\
& =-1
\end{aligned}
$$

with equality if and only if $\left\langle\lambda+\delta, \kappa_{m_{a}}\right\rangle=\left\langle\lambda+\delta, \kappa_{m_{b}}\right\rangle=-s_{m},\left\langle\lambda+\delta, c_{m}\right\rangle=s_{m}$ and $\langle\lambda+\delta, \kappa\rangle=-1$ so that $\left\langle\lambda+\delta, \beta_{n-1}\right\rangle=\left\langle\lambda+\delta, \beta_{n}\right\rangle=-1$.

Next suppose that the tail is compact. If $\left\langle\lambda+\delta, \beta_{\mathfrak{p}_{m}}\right\rangle \leq-2 s_{m}-4$, then we have

$$
\begin{aligned}
\left\langle\lambda+\delta, P_{m}\right\rangle & =\langle\lambda+\delta, \kappa\rangle-\left\langle\lambda+\delta, \beta_{\mathfrak{p}_{m}-1}+\beta_{\mathfrak{p}_{m}}\right\rangle \\
& \geq-\left(2 s_{m}+3\right)-1+2 s_{m}+4 \quad \text { by Lemma 3.1, (AS5) } \\
& =0
\end{aligned}
$$

If $\left\langle\lambda+\delta, \beta_{\mathfrak{p}_{m}}\right\rangle \geq-2 s_{m}-3$ then we compute

$$
\begin{aligned}
\left\langle\lambda+\delta, P_{m}\right\rangle & =\left\langle\lambda+\delta, \beta_{\mathfrak{p}_{m}}+2 c_{m}+\beta_{n-1}+\beta_{n}\right\rangle \\
& \geq-2 s_{m}-3+2 s_{m}+2 \\
& =-1
\end{aligned}
$$

with equality if and only if $\left\langle\lambda+\delta, \beta_{\mathfrak{p}_{m}}\right\rangle=-2 s_{m}-3,\left\langle\lambda+\delta, c_{m}\right\rangle=s_{m}$ and $\left\langle\lambda+\delta, \beta_{n-1}\right\rangle=\left\langle\lambda+\delta, \beta_{n}\right\rangle=1$. This completes the proof of Proposition 5.6.

As an immediate consequence of Proposition 5.6 and integrality, we have

Corollary 5.7. Except in the cases of equality in Proposition 5.6, Theorem 4.6 applies so that we have the conclusion of Theorem 4.9. 
Proof of Theorem 4.9. By Corollary 5.7, we are only left to consider Vogan diagrams as in Table 4. First suppose that $\alpha=\beta_{\mathfrak{p}_{i}}$ is the noncompact simple root chosen by the Knapp process. Consider the roots $\gamma \in C$ listed in Table 2; we need to show $\left\langle\lambda+\delta, s_{\alpha} \gamma\right\rangle \geq 0$ for these roots.

Lemma 3.2 handles the $\gamma_{j}^{A}$. Next consider the roots $\gamma_{j} \in C$. Here $\left|\beta_{\mathfrak{p}_{i}}\right|^{2}=$ $\left|\gamma_{j}\right|^{2}$ so that $s_{\alpha} \gamma_{j}=\gamma_{j}-\beta_{\mathfrak{p}_{i}}$. For $i<j \leq m$, we compute

$$
\begin{aligned}
& \left\langle\lambda+\delta, \gamma_{j}-\beta_{\mathfrak{p}_{i}}\right\rangle \\
& =\left\langle\lambda+\delta, \gamma_{j-1}^{A}-\beta_{\mathfrak{p}_{i}}\right\rangle \\
& \quad+\sum_{x=j}^{m}\left\langle\lambda+\delta, c_{x-1}+2 \beta_{\mathfrak{p}_{x}}+c_{x}\right\rangle+\langle\lambda+\delta, E\rangle \\
& \quad \geq \sum_{x=j}^{m}\left\langle\lambda+\delta, c_{x-1}+2 \beta_{\mathfrak{p}_{x}}+c_{x}\right\rangle+\langle\lambda+\delta, E\rangle \quad \text { by Lemma } 3.2 \\
& \geq 0 \quad \text { by Lemma } 5.3 .
\end{aligned}
$$

For $j=i$, we have $\gamma_{i}=\beta_{\mathfrak{p}_{i}-1}+\beta_{\mathfrak{p}_{i}}+P_{i}$. If $\beta_{\mathfrak{p}_{i}-1}$ is compact then $\left\langle\lambda+\delta, \beta_{\mathfrak{p}_{i}-1}\right\rangle \geq 1$ by Lemma 3.1. If $\beta_{\mathfrak{p}_{i}-1}$ is noncompact then

$$
\left\langle\lambda+\delta, \beta_{\mathfrak{p}_{i}-1}\right\rangle=\left\langle\lambda+\delta, \kappa_{i-1}-\beta_{\mathfrak{p}_{i}}\right\rangle \geq\left\langle\lambda+\delta,-\beta_{\mathfrak{p}_{i}}\right\rangle \geq 1
$$

by Lemma 3.1 and (P2). Combining this with Proposition 5.6 and Lemma 3.3, we have

$$
\left\langle\lambda+\delta, \gamma_{i}-\beta_{\mathfrak{p}_{i}}\right\rangle=\left\langle\lambda+\delta, \beta_{\mathfrak{p}_{i}-1}+P_{i}\right\rangle \geq 1+\left\langle\lambda+\delta, P_{i}\right\rangle \geq 0,
$$

for the desired result for $\gamma_{i}$.

For $1 \leq j<i$, we consider $\gamma_{j}=\gamma_{j}^{A}+P_{i}$. Lemma 3.2 and Proposition 5.6 combine to give

$\left\langle\lambda+\delta, \gamma_{j}-\beta_{\mathfrak{p}_{i}}\right\rangle=\left\langle\lambda+\delta, \gamma_{j}^{A}-\beta_{\mathfrak{p}_{i}}\right\rangle+\left\langle\lambda+\delta, P_{i}\right\rangle \geq 0+\left\langle\lambda+\delta, P_{m}\right\rangle \geq-1$.

If we can show that the above inequality is strict, then integrality will provide the result for $\gamma_{j}$. So, assume (5.19) is an equality so that $\left\langle\lambda+\delta, \gamma_{j-1}^{A}-\beta_{\mathfrak{p}_{i}}\right\rangle=0$ and $\left\langle\lambda+\delta, P_{i}\right\rangle=\left\langle\lambda+\delta, P_{m}\right\rangle=-1$. From Lemmas 3.2, 3.3, and Proposition 5.6 , this in turn implies

(1) $\left\langle\lambda+\delta, c_{x}\right\rangle=s_{x}$ for $j \leq x \leq m$,

(2) $\left\langle\lambda+\delta, \kappa_{x}\right\rangle=-s_{x}$ for $j \leq x \leq m-1$,

(3) the remaining conditions in Table 4 , and

(4) $\left\langle\lambda+\delta, \beta_{\mathfrak{p}_{j}}\right\rangle=\left\langle\lambda+\delta, \beta_{\mathfrak{p}_{i}}\right\rangle$. 
We show conditions (1)-(3), along with the fact that $A_{\mathfrak{q}}(\lambda)$ is weakly fair, contradict (4). To do so, we prove the following claim.

CLAIM 5.8. If (1)-(3) hold, and $A_{\mathfrak{q}}(\lambda)$ is weakly fair, then

$$
\left\langle\lambda+\delta, \beta_{\mathfrak{p}_{x-1}}-\beta_{\mathfrak{p}_{x}}\right\rangle \leq-2 \quad \text { for } \quad j+1 \leq x \leq m .
$$

Proof of Claim. The proof proceeds by downward induction. First note that for $j+1 \leq x \leq m$, conditions (1) and (2) imply

$$
\left\langle\lambda+\delta, \beta_{\mathfrak{p}_{x-1}}-\beta_{\mathfrak{p}_{x}}\right\rangle=-2 s_{x-1}-2\left\langle\lambda+\delta, \beta_{\mathfrak{p}_{x}}\right\rangle .
$$

For the base step, $x=m$, first suppose $\beta_{n}$ is noncompact. Then Lemma 5.2 shows that $-2 s_{m-1} \leq 4\left\langle\lambda+\delta, \beta_{\mathfrak{p}_{m}}\right\rangle+2 s_{m}$ so that (5.19) becomes

$$
\left\langle\lambda+\delta, \beta_{\mathfrak{p}_{m-1}}-\beta_{\mathfrak{p}_{m}}\right\rangle \leq 2\left\langle\lambda+\delta, \beta_{\mathfrak{p}_{m}}\right\rangle+2 s_{m} .
$$

In the $B_{n}$ case, Proposition 5.6 implies $\left\langle\lambda+\delta, \beta_{\mathfrak{p}_{m}}\right\rangle=-2 s_{m}$. Moreover, Lemma 5.2 and $\left\langle\lambda+\delta, \beta_{n}\right\rangle=-\frac{1}{2}$, show that $s_{m} \geq 1$. Therefore, from (5.20) we deduce the base step.

In the $C_{n}$ case, $\left\langle\lambda+\delta, \beta_{\mathfrak{p}_{m}}\right\rangle=-\left\langle\lambda+\delta, \beta_{n}\right\rangle-2 s_{m}-1$. Moreover, Lemma 5.2 implies $-2\left\langle\lambda+\delta, \beta_{n}\right\rangle \leq 2 s_{m}$. Therefore, from (5.21) we deduce the base step.

In the $D_{n}$ case, Proposition 5.6 implies $\left\langle\lambda+\delta, \beta_{\mathfrak{p}_{m}}\right\rangle=-2 s_{m}+1$. Moreover, Lemma 5.2 and $\left\langle\lambda+\delta, \beta_{n}\right\rangle=-1$, show that $s_{m} \geq 2$. Therefore, from (5.20) we deduce the base step.

Now suppose that $\beta_{n}$ is compact. In the $B_{n}$ case, Proposition 5.6 implies $\left\langle\lambda+\delta, \beta_{\mathfrak{p}_{m}}\right\rangle=-2\left(s_{m}+1\right)$. Moreover, Lemma 5.2 implies $-2 s_{m-1} \leq 4\langle\lambda+$ $\left.\delta, \beta_{\mathfrak{p}_{m}}\right\rangle+4 s_{m}+2$. Therefore, from (5.19) we deduce the base step.

In the $D_{n}$ case, Proposition 5.6 implies $\left\langle\lambda+\delta, \beta_{\mathfrak{p}_{m}}\right\rangle=-\left(2 s_{m}+3\right)$. Moreover, Lemma 5.2 implies $-2 s_{m-1} \leq 4\left\langle\lambda+\delta, \beta_{\mathfrak{p}_{m}}\right\rangle+4 s_{m}+4$. Therefore, from (5.19) we deduce the base step.

Now suppose that the claim holds for $x=k+1$. Then we compute

$$
\begin{array}{rlr}
\left\langle\lambda+\delta, \beta_{\mathfrak{p}_{k-1}}-\beta_{\mathfrak{p}_{k}}\right\rangle & =-2 s_{k-1}-2 \beta_{\mathfrak{p}_{k}} & \\
& \leq 2\left\langle\lambda+\delta, \beta_{\mathfrak{p}_{k}}\right\rangle+2 s_{k} & \text { by Lemma } 5.2 \\
& =\left\langle\lambda+\delta, \beta_{\mathfrak{p}_{k}}\right\rangle-\left\langle\lambda+\delta, \beta_{\mathfrak{p}_{k+1}}\right\rangle & \text { by (1) and (2) } \\
& \leq-2 \quad \text { by inductive hypothesis. } &
\end{array}
$$

This completes the proof of the claim.

The claim contradicts (4) so we may conclude that (5.18) is an inequality. Therefore we have the desired result for $\gamma_{j}$ when $1 \leq j<i$. 
Continuing through Table 2, now consider $\gamma=\gamma^{S}=\gamma_{m}^{A}+c_{m}+T$ where $T=\beta_{n}$ in $B_{n}$ or $C_{n}$, and $T=\beta_{n-1}+\beta_{n}$ in $D_{n}$. In each case, $s_{\alpha}\left(\gamma^{S}\right)=\gamma^{S}-\alpha$. Therefore

$$
\begin{aligned}
\left\langle\lambda+\delta, s_{\alpha}\left(\gamma^{S}\right)\right\rangle & =\left\langle\lambda+\delta, \gamma_{m}^{A}-\alpha\right\rangle+\left\langle\lambda+\delta, c_{m}+T\right\rangle \\
& \geq s_{m}+\langle\lambda+\delta, T\rangle
\end{aligned}
$$

by Lemmas 3.1 and 3.2. If the diagram has a noncompact tail, then (5.21) and Lemma 5.2 show $\left\langle\lambda+\delta, s_{\alpha}\left(\gamma^{S}\right)\right\rangle \geq 0$; if the diagram has a compact tail, then (5.21) and Lemma 3.1 show $\left\langle\lambda+\delta, s_{\alpha}\left(\gamma^{S}\right)\right\rangle>0$.

To finish the argument in the case that $\alpha=\beta_{\mathfrak{p}_{i}}$, we need to check the $B_{n}$ root $\gamma^{S+}$ and the $C_{n}$ root $\gamma_{i}^{\prime}$. In the $B_{n}$ case, similiar to $\gamma^{S}$, we find

$$
\left\langle\lambda+\delta, s_{\alpha}\left(\gamma^{S+}\right)\right\rangle \geq s_{m}+\left\langle\lambda+\delta, \beta_{n}\right\rangle,
$$

so that in the noncompact tail case, Lemma 5.2 implies the result and in the compact tail case, Lemma 3.1 implies the result. For $C_{n}$, we compute

$$
\left\langle\lambda+\delta, s_{\alpha}\left(\gamma_{i}^{\prime}\right)\right\rangle=\left\langle\lambda+\delta, \gamma_{i}^{\prime}-2 \beta_{\mathfrak{p}_{i}}\right\rangle=\left\langle\lambda+\delta, P_{i}-\beta_{\mathfrak{p}_{i}}\right\rangle \geq-1+1=0
$$

by Proposition 5.6 and (P2).

Now suppose $\alpha=\beta_{n}$, so that the Vogan diagram has a noncompact tail. We show that $\left\langle\lambda+\delta, P_{m}\right\rangle \geq 0$ so that Theorem 4.6 gives the result. To do so, we compute

$$
\begin{aligned}
& \left\langle\lambda+\delta, P_{m}\right\rangle=\left\langle\lambda+\delta, \beta_{\mathfrak{p}_{m}}+c_{m}+E\right\rangle \\
& \geq\left\langle\lambda+\delta, \beta_{\mathfrak{p}_{m}}+c_{m}\right\rangle \\
& \text { by Lemma } 5.3 \\
& \geq\left\langle\lambda+\delta, \beta_{\mathfrak{p}_{m}}\right\rangle+s_{m} \\
& \geq \begin{cases}s_{m}+2\left\langle\lambda+\delta, \beta_{n}\right\rangle & \text { for } B_{n} \text { diagrams } \\
s_{m}+\frac{1}{2}\left\langle\lambda+\delta, \beta_{n}\right\rangle & \text { for } C_{n} \text { diagrams } \\
s_{m}+\left\langle\lambda+\delta, \beta_{n}\right\rangle & \text { for } D_{n} \text { diagrams }\end{cases} \\
& \geq \begin{cases}s_{m}+2\left(-\frac{1}{2} s_{m}\right) & \text { for } B_{n} \text { diagrams } \\
s_{m}+\frac{1}{2}\left(-s_{m}\right) & \text { for } C_{n} \text { diagrams } \\
s_{m}+-\frac{1}{2} s_{m} & \text { for } D_{n} \text { diagrams }\end{cases} \\
& \geq 0 \text {. }
\end{aligned}
$$

This completes the proof of Theorem 4.9. 


\section{REFERENCES}

1. Flensted-Jensen, M., Discrete series for semisimple symmetric spaces, Ann. of Math. 111 (1980), 253-311.

2. Friedman, P., The Langlands Parameters of Subquotients of Certain Derived Functor Modules, J. Funct. Anal. 157 (1998), 210-241.

3. Knapp, A., Representation Theory of Semisimple Groups: An Overview Based on Examples, Princeton University Press, Princeton, 1986.

4. Knapp, A., Exceptional Unitary Representations, Representation Theory, an Electronic Journal of the AMS 1 (1997), 1-24.

5. Knapp, A., Lie Groups Beyond an Introduction, Birkhäuser, Boston, 1996.

6. Knapp, A. and Vogan, D., Unitary Representations and Cohomological Induction, Princeton University Press, Princeton, 1995.

7. Knapp, A. and Zuckerman, G., Classification of irreducible tempered representations of semisimple groups, Ann. of Math. 116 (1982), 389-501.

8. Langlands, R. P., On the classification of irreducible representations of real algebraic groups in preprint, Institute for Advanced Study, Princeton (1973), reprinted in Representation Theory and Harmonic Analysis on Semisimple Lie Groups, (P. Sally and D. Vogan, eds.), Math. Surveys Monographs (1989), 101-170.

9. Schlichtkrull, H., The Langlands parameters of Flensted-Jensen's discrete series for semisimple symmetric spaces, J. Funct. Anal. 50 (1983), 133-150.

10. Schlichtkrull, H., On Some Series of Representations Related to Symmetric Spaces, Soc. Math. France 15 (1984), 277-289.

11. Vogan, D., Unitarizability of certain series of representations, Ann. of Math. 120 (1984), 141-187.

12. Wallach, N., On the unitarizability of derived functor modules, Invent. Math. 78 (1984), $131-141$.

DEPARTMENT OF MATHEMATICS

UNION COLLEGE

SCHENECTADY, NY 12308

USA

E-mail: friedmap@union.edu 